Article

\title{
Molecular Classification of Pesticides Including Persistent Organic Pollutants, Phenylurea and Sulphonylurea Herbicides
}

\author{
Francisco Torrens $^{1, *}$ and Gloria Castellano ${ }^{2}$ \\ 1 Institut Universitari de Ciència Molecular, Universitat de València, Edifici d'Instituts de Paterna, \\ P.O. Box 22085, E-46071 València, Spain \\ 2 Facultad de Veterinaria y Ciencias Experimentales, Universidad Católica de Valencia San Vicente \\ Mártir, Guillem de Castro-94, E-46001 València, Spain; E-Mail: gloria.castellano@ucv.es \\ * Author to whom correspondence should be addressed; E-Mail: torrens@uv.es; \\ Tel.: +34-963-544-431; Fax: +34-963-543-274.
}

Received: 8 April 2014; in revised form: 29 May 2014 / Accepted: 30 May 2014 /

Published: 5 June 2014

\begin{abstract}
Pesticide residues in wine were analyzed by liquid chromatography-tandem mass spectrometry. Retentions are modelled by structure-property relationships. Bioplastic evolution is an evolutionary perspective conjugating effect of acquired characters and evolutionary indeterminacy-morphological determination-natural selection principles; its application to design co-ordination index barely improves correlations. Fractal dimensions and partition coefficient differentiate pesticides. Classification algorithms are based on information entropy and its production. Pesticides allow a structural classification by nonplanarity, and number of $\mathrm{O}, \mathrm{S}, \mathrm{N}$ and $\mathrm{Cl}$ atoms and cycles; different behaviours depend on number of cycles. The novelty of the approach is that the structural parameters are related to retentions. Classification algorithms are based on information entropy. When applying procedures to moderate-sized sets, excessive results appear compatible with data suffering a combinatorial explosion. However, equipartition conjecture selects criterion resulting from classification between hierarchical trees. Information entropy permits classifying compounds agreeing with principal component analyses. Periodic classification shows that pesticides in the same group present similar properties; those also in equal period, maximum resemblance. The advantage of the classification is to predict the retentions for molecules not included in the categorization. Classification extends to phenyl/sulphonylureas and the application will be to predict their retentions.
\end{abstract}


Keywords: periodic law; periodic property; periodic table; molecular classification

\section{Introduction}

Twenty-six billion litres of wine were produced worldwide and 24 billion litres, consumed in 2010 according to the International Organization of Vine and Wine. Wine, especially red wine, is rich in polyphenols (e.g., resveratrol, catechin, epicatechin), which are antioxidants that protect cells from oxidative damage caused by free radicals. Red-wine antioxidants inhibit cancer development, e.g., that of prostate cancer. Red-wine consumption presents heart-health benefits. Application of pesticides (e.g., fungicides, insecticides) to improve grape yields is common. However, pesticides permeate via the plant tissues and remain in harvested grapes/processed products (e.g., grape juice, wine). Because pesticides are a source of toxicants that are harmful to human beings it is important to test for their levels in grapes, juice and wine. Although EU has set maximum residue levels (MRLs) for pesticides in wine grapes of $0.01-10 \mathrm{mg} \cdot \mathrm{kg}^{-1}$ it has not done so for wine. An EU-wine study revealed that 34 out of 40 bottles contained at least one pesticide. Average number was $>4$ pesticides per bottle while the highest number was 10 . Pesticide analysis in red wine is challenging because of the complexity of the matrix that contains alcohol, organic acids, sugars, phenols and pigments, e.g., anthocyanins. Traditional red-wine sample preparation methods include liquid-liquid extraction (LLE) with organic solvents [1,2] and solid-phase extraction (SPE) with reversed-phase C18/polymeric sorbents [3-5]. However, LLE is labour-intensive, consumes large amounts of organic solvents and forms emulsions making difficult to separate organic/aqueous phases. In contrast, SPE demands more development. Solid-phase microextraction (SPME) [6,7], hollow-fibre liquid-phase microextraction [8] and stir-bar sorptive extraction (SBSE) [9] are lesser reproducible. Typical detections incorporate gas chromatography (GC), GC coupled to mass spectrometry (MS) (GC-MS) and liquid chromatography coupled to tandem MS (LC-MS-MS).

Quick, easy, cheap, effective, rugged and safe (QuEChERS) is a sample preparation method that was reported for pesticide-residue determination in vegetables/fruits [10]; it was used for pesticide/compound analysis in various food, oil and beverage matrices [11-13]; QuEChERS involves pesticide extraction from a sample with high water content into acetonitrile, with addition of salts to separate phases and partition the pesticides into the organic layer, which is followed by dispersive SPE (dSPE) to clean up various matrix co-extractives and achieve mixing of an aliquot of sample extract with sorbents prepacked in a centrifuge tube. Pesticide determination in red wine was reported [14]. Eight pesticides belonging to the insecticide (methamidophos, diazinone, pyrazophos, chlorpyrifos), fungicide (carbendazim, thiabendazole, pyrimethanil, cyprodinil, pyrazophos) and parasiticide (thiabendazole) classes were selected. Their polarities are different. Some are planar (carbendazim, thiabendazole, pyrimethanil, cyprodinil). Cyprodinil was most usually detected on grapes with chlorpyrifos, diazinone and methamidophos, frequent. Carbendazim was detected in three out of six red-wine samples. Occurrence and removal efficiency of pesticides in sewage treatment plants from Spanish, Mediterranean, Brazilian and other rivers were reviewed [15,16] and reported [17,18]. Transport of organic persistent microcontaminants associated with suspended particulate material in 
the Ebro River Basin was described [19,20]. Several researchers have reported the quantitative structure-activity/property relationships (QSAR/QSPR) of pesticides. The Benfenati group modelled the QSPR of the octanol/water partition coefficient of organometallic substances by optimal SMILESbased descriptors [21], QSAR of the toxicity of organic substances to Daphnia magna via freeware CORAL [22], and optimal descriptor as a translator of eclectic data into endpoint prediction and mutagenicity of fullerene as a mathematical function of conditions [23]. The Roy group modelled predictive chemometrics and three-dimensional toxicophore mapping of diverse organic chemicals causing bioluminescent repression of the bacterium genus Pseudomonas [24] and QSAR for toxicity of ionic liquids to D. magna analyzing aromaticity $v$ s. lipophilicity [25].

The chromatographic retention time was correlated to the stationary and mobile phases of the system. In earlier publications the free energy of solvation and partition coefficients in methanol-water binary mixtures were analized [26]. Stationary phase was modelled in size-exclusion chromatography with binary eluents as a strategy in size-exclusion chromatography [27]. Stationary-mobile phase distribution coefficient for polystyrene standards was represented [28]. A new chemical index inspired by plastic evolution was presented [29] and applied to valence-isoelectronic series of aromatics [30]. QSPR of retention times of phenylureas [31,32] and pesticides [33] was described by plastic evolution. A simple computerized algorithm was proposed to be useful for establishing relationships between chemical structures and biosignificance [34,35]. Starting point is to use information entropy for pattern recognition. Entropy is formulated on basis of similarity matrix between two biochemical species. As entropy is weakly discriminating for classification, the more powerful concepts of entropy production and equipartition conjecture were introduced [36]. The aim of the present report is to find properties that distinguish pesticide structures according to retention times. The study applies a chemical index to pesticides. The goal is index usefulness validation via the capability to distinguish between pesticides, and interest as a predictive index for retention as compared with fractal dimensions and partition coefficients. Section 2 illustrates and discusses the results. Section 3 presents the computational method, including classification algorithm, information entropy, equipartition conjecture of entropy production and learning procedure. Finally, the last section summarizes our conclusions.

\section{Results and Discussion}

For pesticides, LC-MS-MS retention times $R_{\mathrm{t}}$ were taken from Wang and Telepchak. Methamidophos was taken as the reference $R_{\mathrm{t}}\left(R_{\mathrm{t}}{ }^{\circ}\right)$ because of its least $R_{\mathrm{t}}(c f$. Table 1). Internal standard (IS) triphenyl phosphate (TPP) was included in the classification. The $\left(R_{\mathrm{t}}-R_{\mathrm{t}}^{\circ}\right) / R_{\mathrm{t}}{ }^{\circ}$ ratios were calculated. Molecular fractal dimensions were computed with our program TOPO [37].

Variations of $\left(R_{\mathrm{t}}-R_{\mathrm{t}}{ }^{\circ}\right) / R_{\mathrm{t}}{ }^{\circ} v s$. 1-octanol-water partition coefficient and fractal dimension averaged for nonburied atoms minus molecular fractal dimension $D^{\prime}-D$ show fit. The regression turns out to be:

$$
\begin{gathered}
\left(R_{t}-R_{t}^{o}\right) / R_{t}^{o}=-0.188+0.367 \log P+19.6\left(D^{\prime}-D\right), n=9, r=0.973, s=0.337, F=53.3 \\
\operatorname{MAPE}=8.77 \% \mathrm{AEV}=0.0533
\end{gathered}
$$

where mean absolute percentage error (MAPE) is $8.77 \%$ and approximation error variance (AEV), 0.0533. If IS TPP is excluded the results are improved: 


$$
\begin{gathered}
\left(R_{t}-R_{t}^{o}\right) / R_{t}^{o}=-0.229+0.420 \log P+19.1\left(D^{\prime}-D\right), n=8 r=0.982 s=0.295 \\
F=67.5, \mathrm{MAPE}=7.17 \% \mathrm{AEV}=0.0357
\end{gathered}
$$

and AEV decays by $33 \%$. When $D$ ' is included in the fit the correlation is bettered:

$$
\begin{gathered}
\left(R_{t}-R_{t}^{o}\right) / R_{t}^{o}=-11.6+0.272 \log P+9.44 D^{\prime}, n=8 \quad r=0.987 s=0.253 \quad F=93.2 \\
\text { MAPE }=5.81 \% \quad \mathrm{AEV}=0.0261
\end{gathered}
$$

and AEV drops by $51 \%$. The best quadratic model vs. $D$ ' improves the fit:

$$
\begin{gathered}
\left(R_{t}-R_{t}^{o}\right) / R_{t}^{o}=-112+151 D^{\prime}-49.3 D^{\prime 2}, n=9 \quad r=0.988 s=0.224 \quad F=125.2 \\
\mathrm{MAPE}=5.39 \% \quad \mathrm{AEV}=0.0234
\end{gathered}
$$

and AEV decreases by $56 \%$. If IS TPP is excluded the results are bettered:

$$
\begin{gathered}
\left(R_{t}-R_{t}^{o}\right) / R_{t}^{o}=-107+144 D^{\prime}-46.7 D^{\prime 2}, n=8 \quad r=0.989 s=0.228 \quad F=114.4 \\
\text { MAPE }=5.84 \% \quad \text { AEV }=0.0214
\end{gathered}
$$

and AEV decays by 60\%. Model (3) is linear and expected to perform better than Equations (4) and (5) for extrapolation. However, the latter are nonlinear and could function better than Equation (3) for

\begin{tabular}{|c|c|c|c|c|c|c|c|}
\hline Compound & $\begin{array}{c}R_{\mathrm{t}} \\
(\mathrm{min})\end{array}$ & $\begin{array}{c}R_{\mathrm{t}}-\boldsymbol{R}_{\mathrm{t}}^{\circ} \\
(\mathrm{min})\end{array}$ & $\left(R_{\mathrm{t}}-\boldsymbol{R}_{\mathrm{t}}^{\circ}\right) / \boldsymbol{R}_{\mathrm{t}}^{\circ}$ & $\log P$ & $\mathrm{p} K_{\mathrm{a}}$ & D & D' \\
\hline 1. Methamidophos $\mathrm{C}_{2} \mathrm{H}_{8} \mathrm{NO}_{2} \mathrm{PS}<001010>$ & 2.78 & 0.00 & 0.00000 & -0.779 & -0.58 & 1.235 & 1.266 \\
\hline 2. Carbendazim $\mathrm{C}_{9} \mathrm{H}_{9} \mathrm{~N}_{3} \mathrm{O}_{2}<100010>$ & 6.48 & 3.70 & 1.33094 & 1.52 & 5.66 & 1.284 & 1.332 \\
\hline 3. Thiabendazole $\mathrm{C}_{10} \mathrm{H}_{7} \mathrm{~N}_{3} \mathrm{~S}<110010>$ & 6.91 & 4.13 & 1.48561 & 2.47 & 3.40 & 1.288 & 1.331 \\
\hline 4. Pyrimethanil $\mathrm{C}_{12} \mathrm{H}_{13} \mathrm{~N}_{3}<110010>$ & 10.43 & 7.65 & 2.75180 & 2.558 & 4.41 & 1.314 & 1.407 \\
\hline 5. Cyprodinil $\mathrm{C}_{14} \mathrm{H}_{15} \mathrm{~N}_{3}<110010>$ & 11.44 & 8.66 & 3.11511 & 3.012 & 4.22 & 1.344 & 1.470 \\
\hline 6. TPP (IS) $\mathrm{C}_{18} \mathrm{H}_{15} \mathrm{O}_{4} \mathrm{P}<111000>$ & 11.78 & 9.00 & 3.23741 & 4.63 & -5 & 1.394 & 1.504 \\
\hline 7. Diazinone $\mathrm{C}_{12} \mathrm{H}_{21} \mathrm{~N}_{2} \mathrm{O}_{3} \mathrm{PS}<111100>$ & 11.92 & 9.14 & 3.28777 & 3.766 & 1.21 & 1.398 & 1.509 \\
\hline 8. Pyrazophos $\mathrm{C}_{14} \mathrm{H}_{20} \mathrm{~N}_{3} \mathrm{O}_{5} \mathrm{PS}<111110>$ & 12.24 & 9.46 & 3.40288 & 2.810 & -1.37 & 1.403 & 1.505 \\
\hline 9. Chlorpyrifos $\mathrm{C}_{9} \mathrm{H}_{11} \mathrm{NO}_{3} \mathrm{PSCl}_{3}<111111>$ & 13.42 & 10.64 & 3.82734 & 5.004 & -5.28 & 1.394 & 1.494 \\
\hline
\end{tabular}
intrapolation. Additional fitting parameters were tested: absolute/differential formation enthalpies, molecular dipole moment, organic solvent/water partition coefficients, free energies of solvation and water $\rightarrow$ organic solvent transfer, molecular volume, surface area, globularity, rugosity, hydrophobic, hydrophilic and total solvent accessible surfaces, and numbers of $\mathrm{P}$ and total atoms. However, the results do not improve Equations (3)-(5).

Table 1. Vector property ( $\left.\operatorname{cyc}_{123}, \mathrm{O}_{0345}, \mathrm{NP}, \mathrm{S}=, \mathrm{N}_{13}, \mathrm{Cl}_{3}\right)$, retention, $\log P, \mathrm{p} K_{\mathrm{a}}$ and dimensions for pesticides.

Pearson correlation coefficient matrix $\mathbf{R}$ was calculated between pairs of vector properties $<i_{1}, i_{2}, i_{3}$, $i_{4}, i_{5}, i_{6}>$ for nine pesticides. Intercorrelations are illustrated in the partial correlation diagram, which contains high $(r \geq 0.75)$, medium $(0.50 \leq r<0.75)$, low $(0.25 \leq r<0.50)$ and zero $(r<0.25)$ partial autocorrelations. Pairs of molecules with higher partial correlations show similar vector property. However, results should be taken with care, because Entry 9 with constant vector $<111111>$ shows null standard deviation, causing greatest partial correlations $r=1$ with any compound, which is an artefact. With the equipartition conjecture the upper triangle of $\mathbf{R}$ resulted: 


$\mathbf{R}=\left|\begin{array}{lllllllll}0.984 & 0.359 & 0.109 & 0.109 & 0.109 & 0.203 & 0.141 & 0.172 & 0.156 \\ & 0.984 & 0.734 & 0.734 & 0.734 & 0.578 & 0.516 & 0.547 & 0.531 \\ & & 0.984 & 0.984 & 0.984 & 0.828 & 0.766 & 0.797 & 0.781\end{array}\right|$

Some correlations are high, e.g., $R_{3,4}=R_{3,5}=R_{4,5}=0.984$. They are illustrated in the partial correlation diagram, which contains 21 high ( $c f$. Figure 1, red lines), seven medium (orange), one low (yellow) and seven zero (black) partial correlations. Two out of eight high partial correlations of Entry 9 are corrected: its correlation with Entry 2 is medium and its correlation with Entry 1 is zero partial correlation. For instance, pesticide 2 (carbendazim) shows medium partial correlations with molecules 3-9 $(0.50 \leq r<0.75$, orange $)$ and low partial correlation with compound $1(0.25 \leq r<0.50$, yellow).

The grouping rule in the case with equal weights $a_{k}=0.5$ for $b_{1}=0.93$ allows the classes:

$$
\mathrm{C}-b_{1}=(1)(2)(3,4,5)(6)(7,8,9)
$$

Five clusters are obtained with the associated entropy $h-\mathbf{R}-b_{1}=10.70$ matching to $<i_{1}, i_{2}, i_{3}, i_{4}, i_{5}, i_{6}>$ and $\mathrm{C}-b_{1}[38-40]$; the binary taxonomy (Table 1 ) separates the classes $1,2,3,4$ and 5 with 1, 1, 3, 1 and 3 pesticides, respectively [41]. The planar molecules $3-5$ with low retention are grouped into the same class; nonplanar thiophosphates 7-9 with the greatest retention are aggregated into the same cluster. Substances belonging to the same grouping appear highly correlated in the partial correlation diagram (Figure 1). However, $\mathrm{C}-b_{1}$ results should be taken with care because classes (1), (2) and (6) with only one substance could be outliers. At level $b_{2}$ with $0.74 \leq b_{2} \leq 0.76$, the set of groupings turns out to be:

$$
\mathrm{C}-b_{2}=(1)(2)(3,4,5,6,7,8,9)
$$

Three clusters result and entropy decays to $h-\mathbf{R}-b_{2}=3.71$ going with $\left\langle i_{1}, i_{2}, i_{3}, i_{4}, i_{5}, i_{6}>\right.$ and $\mathrm{C}-b_{2}$ dividing classes: 1-3 with 1, 1 and 7 pesticides. Again, nonplanar thiophosphates 7-9 with the greatest retention are aggregated into the same class. Compounds in the same cluster appear highly correlated in partial correlation diagram (Figure 1). Notwithstanding, $\mathrm{C}-b_{2}$ results should be taken with caution because groupings (1) and (2) with a unique compound could be outliers. Table 2 shows comparative analysis of the set containing 1-9 classes in agreement with partial correlation diagram (Figure 1).

From the previous partial correlation diagram (Figure 1) and set of nine classifications (Table 2), we suggest splitting the data into three groupings:

$$
(1,2)(3,4,5)(6,7,8,9)
$$

The pesticides dendrogram ( $c f$. Figure 2 ) shows different behaviour depending on the number of cyles. One more time, the planar molecules 3-5 with low retention are grouped into the same class and nonplanar thiophosphates 6-9 with the greatest retention are aggregated into the same cluster. 
Figure 1. Partial correlation diagram: High (red), medium (orange) and low (yellow) correlations of pesticides.

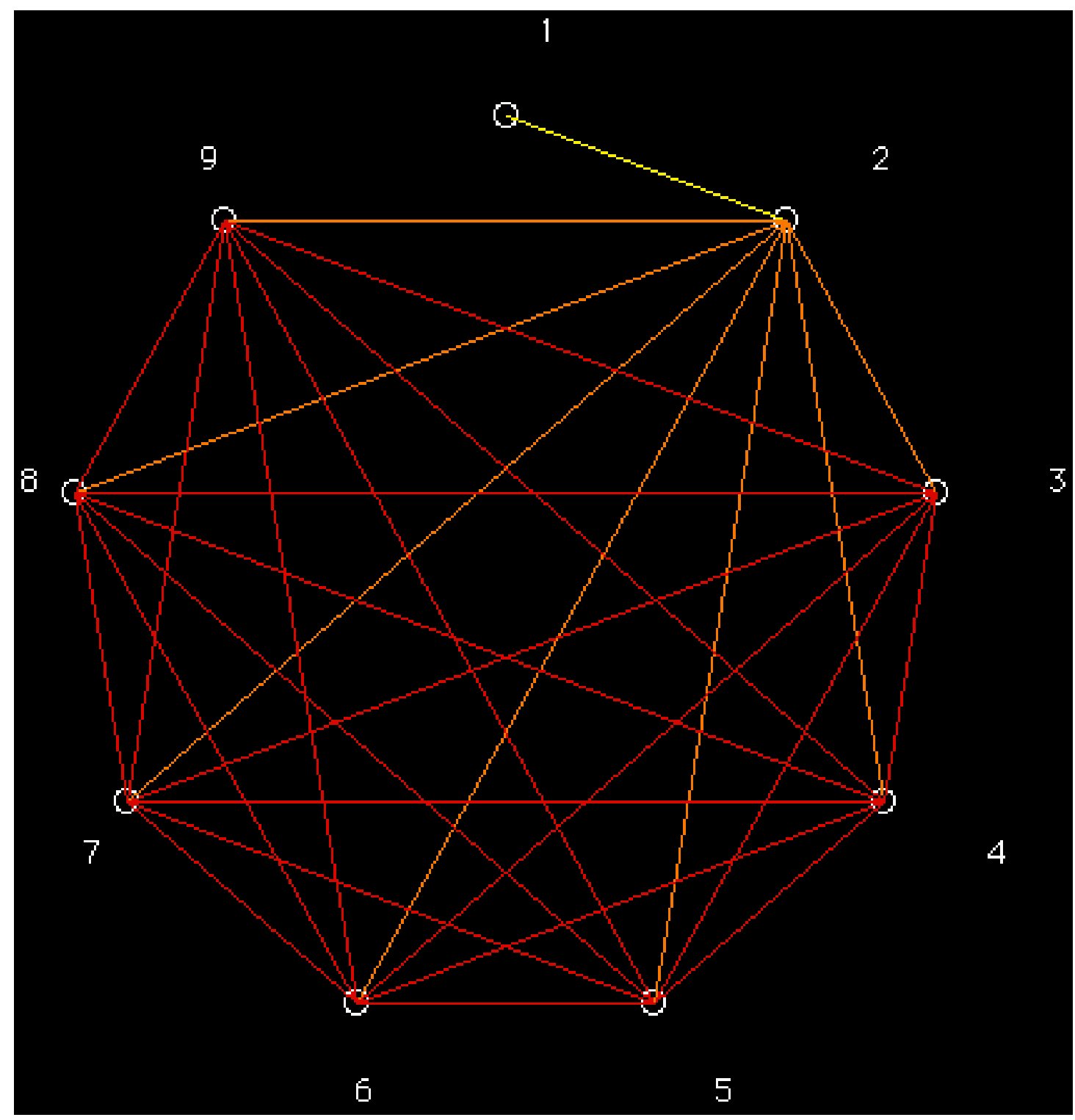

Table 2. Classification level, number of classes and entropy for vector property of pesticides.

\begin{tabular}{ccc}
\hline Classification Level $\boldsymbol{b}$ & Number of Classes & Entropy $\boldsymbol{h}$ \\
\hline 1.00 & 9 & 32.49 \\
0.98 & 7 & 20.01 \\
0.96 & 6 & 15.13 \\
0.93 & 5 & 10.70 \\
0.87 & 4 & 6.77 \\
0.76 & 3 & 3.71 \\
0.51 & 2 & 1.47 \\
0.10 & 1 & 0.08 \\
\hline
\end{tabular}


Figure 2. Dendrogram of pesticides according, top $\rightarrow$ bottom, to: $(1,2)(3,4,5)(6,7,8,9)$.

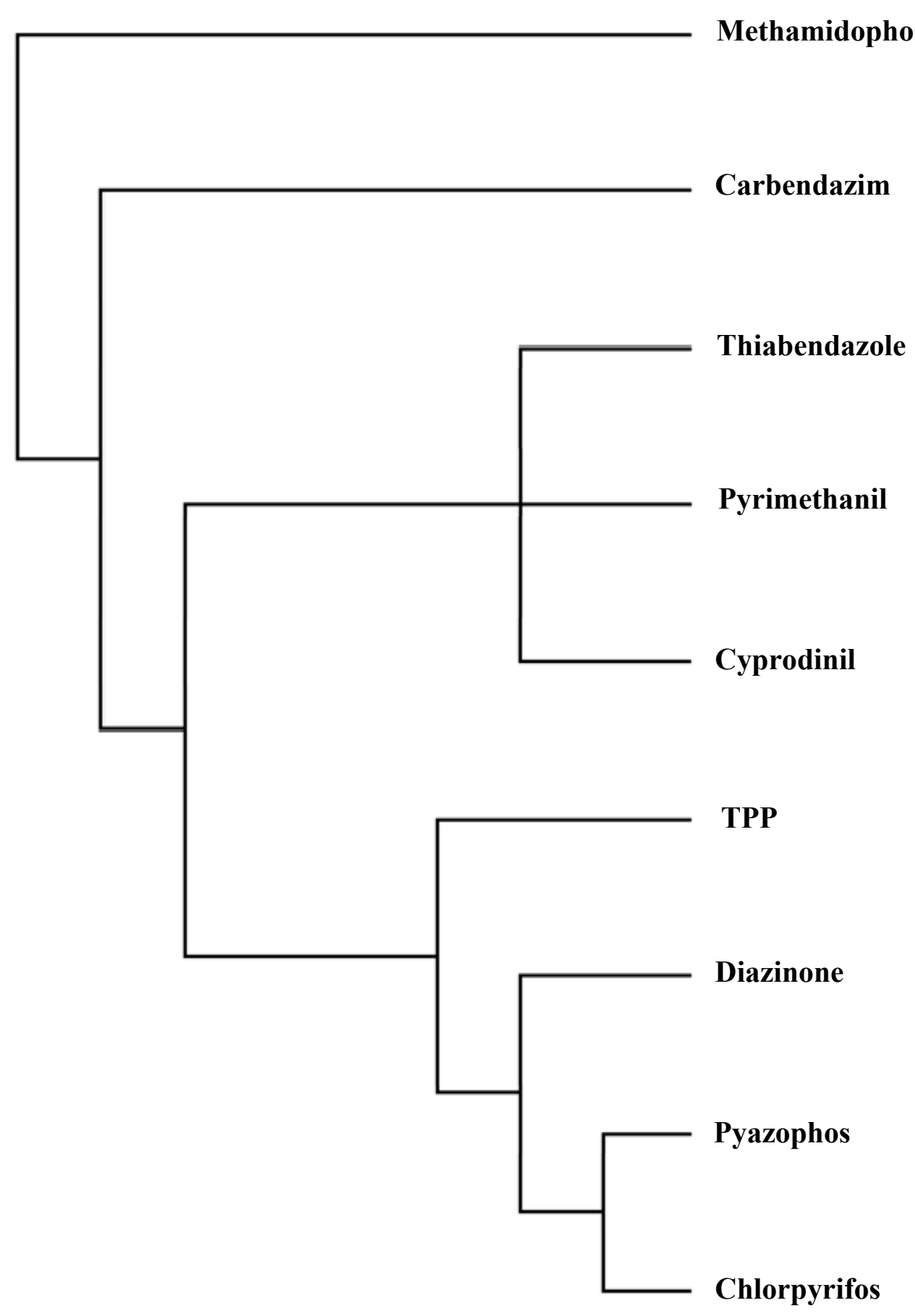

The illustration of the classification above in a radial tree ( $c f$. Figure 3 ) shows the different behaviour of the pesticides depending on the number of cyles. The same classes above are recognized, in qualitative agreement with partial correlation diagram and dendrogram (Figures 1 and 2). One more time, planar molecules 3-5 with low retention are grouped into the same class, and nonplanar thiophosphates 6-9 with the greatest retention are aggregated into identical cluster. 
Figure 3. Radial tree of pesticides according, from top to bottom, to: $(1,2)(3,4,5)(6,7,8,9)$.

\section{Carbendazim}

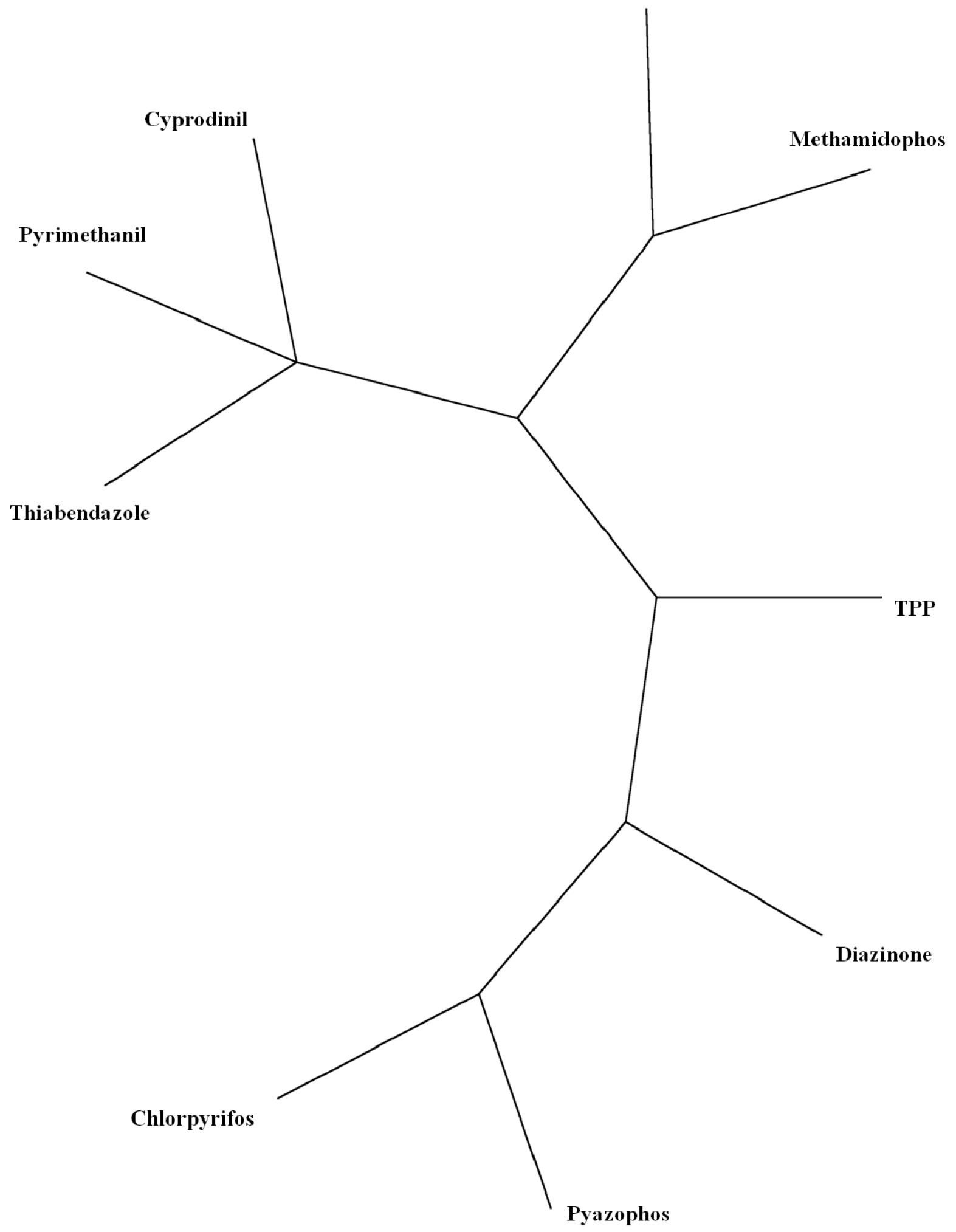

Program SplitsTree allows examining cluster analysis (CA) data [42]. Based on split decomposition it takes as input a distance matrix and produces as output a graph, which represents relations between taxa. For ideal data the graph is a tree whereas less ideal data will give rise to a tree-like net, which is interpreted as possible evidence for conflicting data. As split decomposition does not attempt to force 
data on to a tree it can provide a good indication of how tree-like are given data. In the splits graph for nine pesticides ( $c f$. Figure 4), points 4 and 5 are superimposed on 3, and 9 on 8. It reveals conflicting relationship between class 1 , and groupings 2 and 3 because of interdependences. It indicates spurious relation resulting from base-composition effects. It shows different pesticides behaviour depending on number of cycles in agreement with partial correlation diagram, binary and radial trees (Figures 1-3).

Figure 4. Splits graph of pesticides according, top $\rightarrow$ bottom, to: $(1,2)(3,4,5)(6,7,8,9)$.

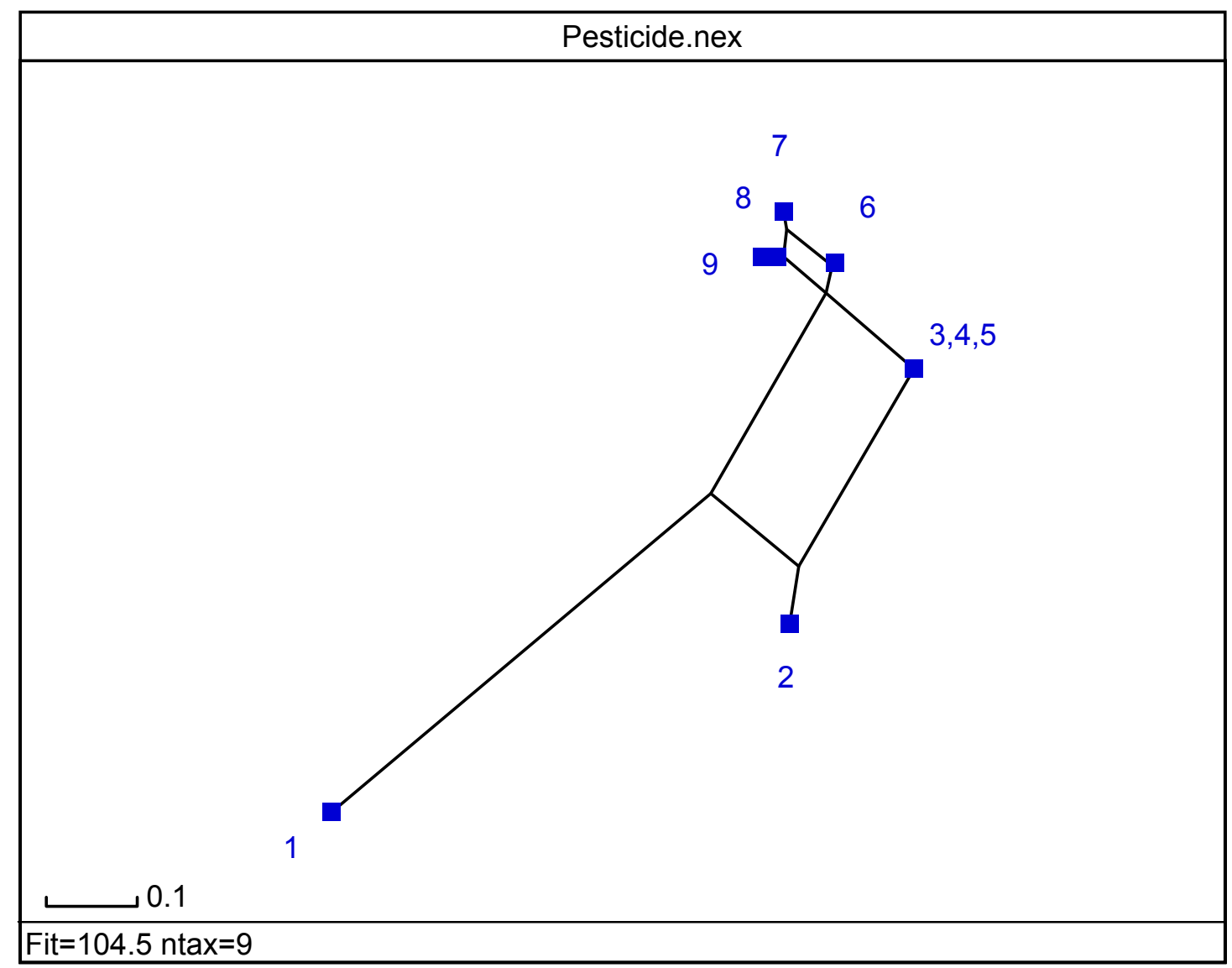

In QSPR, the data file contains less than 100 objects and thousands of $X$-variables. So many $X$-variables exist that no one can discover by inspection patterns, trends, clusters, etc. in objects. Principal components analysis (PCA) is a technique useful to summarize all information contained in $\mathbf{X}$-matrix and put it understandable [43-48]. The PCA works decomposing $\mathbf{X}$-matrix as the product of two smaller matrices $\mathbf{P}$ and $\mathbf{T}$. Loading matrix $(\mathbf{P})$ with information about the variables contains few vectors, the principal components (PCs), which are obtained as linear combinations of the original $X$-variables. In the score matrix $(\mathbf{T})$ with information about objects, every object is described in terms of the projections on to PCs instead of the original variables: $\mathbf{X}=\mathbf{T P}{ }^{\prime}+\mathbf{E}$, where ' denotes the transpose matrix. The information not contained in the matrices remains as unexplained $\mathrm{X}$-variance in the residual matrix $(\mathbf{E})$. Every $\mathrm{PC}_{i}$ is a new co-ordinate expressed as linear combination of old features $x_{j}: \mathrm{PC}_{i}=\sum_{j} b_{i j} x_{j}$. The new co-ordinates $\mathrm{PC}_{i}$ are named scores/factors while the coefficients $b_{i j}$ are called loadings. The scores are ordered according to their information content with regard to the total variance among all objects. Score-score plots show positions of compounds in the new co-ordinate system while loading-loading plots indicate the locations of the features that represent the compounds 
in the new co-ordinates. The PCs present two interesting properties. (1) They are extracted in decaying order of importance. First PC $F_{1}$ always contains more information than the second $F_{2}$ does, $F_{2}$ more than the third $F_{3}$, etc. (2) Every $\mathrm{PC}$ is orthogonal to one another. There is no correlation between the information contained in different PCs. A PCA was performed for the vector properties. The importance of PCA factors $F_{1}-F_{6}$ for $\left\{i_{1}, i_{2}, i_{3}, i_{4}, i_{5}, i_{6}\right\}$ is collected in Table 3 . The use of the first factor $F_{1}$ explains $39 \%$ of variance ( $61 \%$ error), combined application of two factors $F_{1 / 2}$ accounts for $66 \%$ of variance (34\% error), utilization of factors $F_{1-3}$ justifies $87 \%$ of variance ( $13 \%$ error), etc.

Table 3. Importance of the principal component analysis factors for vector property of pesticides.

\begin{tabular}{cccc}
\hline Factor & Eigenvalue & Percentage of Variance & Cumulative Percentage of Variance \\
\hline$F_{1}$ & 2.33109829 & 38.85 & 38.85 \\
$F_{2}$ & 1.62998318 & 27.17 & 66.02 \\
$F_{3}$ & 1.25482746 & 20.91 & 86.93 \\
$F_{4}$ & 0.38517751 & 6.42 & 93.35 \\
$F_{5}$ & 0.33518718 & 5.59 & 98.94 \\
$F_{6}$ & 0.06372637 & 1.06 & 100.00 \\
\hline
\end{tabular}

The PCA factor loadings are shown in Table 4.

Table 4. Principal component analysis loadings for the vector property of pesticides.

\begin{tabular}{ccccccc}
\hline \multirow{2}{*}{ Property } & \multicolumn{5}{c}{ PCA Factor Loadings ${ }^{\text {a }}$} \\
\cline { 2 - 7 } & $\boldsymbol{F}_{\mathbf{1}}$ & $\boldsymbol{F}_{\mathbf{2}}$ & $\boldsymbol{F}_{\mathbf{3}}$ & $\boldsymbol{F}_{\mathbf{4}}$ & $\boldsymbol{F}_{\mathbf{1}}$ & $\boldsymbol{F}_{\mathbf{6}}$ \\
\hline$i_{1}$ & 0.30822766 & 0.64474701 & 0.02673332 & -0.04594797 & 0.50138891 & -0.48485077 \\
$i_{2}$ & 0.44804795 & 0.45046774 & -0.05731613 & 0.08927231 & $\mathbf{- 0 . 7 6 0 0 2 0 3 4}$ & 0.08629170 \\
$i_{3}$ & 0.40956062 & -0.56947041 & -0.18574529 & 0.04102918 & -0.15327503 & -0.66954134 \\
$i_{4}$ & 0.55772042 & -0.17916516 & 0.17892539 & 0.59525799 & 0.32511366 & 0.40595876 \\
$i_{5}$ & -0.31588577 & 0.04253838 & $\mathbf{0 . 7 2 8 5 2 3 1 0}$ & 0.44514023 & -0.20425092 & -0.35748077 \\
$i_{6}$ & 0.35450381 & -0.15222972 & 0.63145758 & -0.66011661 & 0.00822715 & 0.12881324 \\
\hline
\end{tabular}

${ }^{\mathrm{a}}$ Loadings greater than 0.7 are boldfaced.

The PCA $F_{1}-F_{3}$ profile for the vector property is listed in Table 5. For $F_{1}$, variable $i_{4}$ shows the greatest weight in the profile; however, $F_{1}$ cannot be reduced to two variables $\left\{i_{2}, i_{4}\right\}$ without $49 \%$ error. For $F_{2}$, variable $i_{1}$ presents the greatest weight; notwithstanding, $F_{2}$ cannot be reduced to two variables $\left\{i_{1}, i_{3}\right\}$ without $26 \%$ error. For $F_{3}$, variable $i_{5}$ displays the greatest weight; nevertheless, $F_{3}$ cannot be reduced to two variables $\left\{i_{5}, i_{6}\right\}$ without $7 \%$ error. For $F_{4}$, variable $i_{6}$ exhibits the greatest weight; however, $F_{4}$ cannot be reduced to two variables $\left\{i_{4}, i_{6}\right\}$ without $21 \%$ error. For $F_{5}$, variable $i_{2}$ reveals the greatest weight; notwithstanding, $F_{5}$ cannot be reduced to two variables $\left\{i_{1}, i_{2}\right\}$ without $17 \%$ error. For $F_{6}$, variable $i_{3}$ bares the greatest weight; nevertheless, $F_{6}$ cannot be reduced to two variables $\left\{i_{1}, i_{3}\right\}$ without $32 \%$ error. Factors $F_{1-6}$ can be considered as the linear combinations of $\left\{i_{2}, i_{4}\right\},\left\{i_{1}, i_{3}\right\}$, $\left\{i_{5}, i_{6}\right\},\left\{i_{4}, i_{6}\right\},\left\{i_{1}, i_{2}\right\}$ and $\left\{i_{1}, i_{3}\right\}$ with $49 \%, 26 \%, 7 \%, 21 \%, 17 \%$ and $32 \%$ errors. 
Table 5. Profile of the principal component analysis factors for the vector property of pesticides.

\begin{tabular}{ccccccc}
\hline & Percentage of $\boldsymbol{i}_{\mathbf{1}}{ }^{\text {a }}$ & $\mathbf{\%}$ of $\boldsymbol{i}_{\mathbf{2}}$ & $\mathbf{\%}$ of $\boldsymbol{i}_{\mathbf{3}}$ & $\mathbf{\%}$ of $\boldsymbol{i}_{\mathbf{4}}$ & $\mathbf{\%}$ of $\boldsymbol{i}_{\mathbf{5}}$ & $\mathbf{\%}$ of $\boldsymbol{i}_{\mathbf{6}}$ \\
\hline$F_{1}$ & 9.50 & 20.07 & 16.77 & 31.11 & 9.98 & 12.57 \\
$F_{2}$ & 41.57 & 20.29 & 32.43 & 3.21 & 0.18 & 2.32 \\
$F_{3}$ & 0.07 & 0.33 & 3.45 & 3.20 & $\mathbf{5 3 . 0 7}$ & 39.87 \\
$F_{4}$ & 0.21 & 0.80 & 0.17 & 35.43 & 19.81 & 43.58 \\
$F_{5}$ & 25.14 & $\mathbf{5 7 . 7 6}$ & 2.35 & 10.57 & 4.17 & 0.01 \\
$F_{6}$ & 23.51 & 0.74 & 44.83 & 16.48 & 12.78 & 1.66 \\
\hline
\end{tabular}

${ }^{a}$ Percentages greater than $50 \%$ are boldfaced.

In PCA $F_{2}-F_{1}$ scores plot ( $c f$. Figure 5), points 4 and 5 appear superimposed on 3. It shows different behaviour depending on number of cyles. It distinguishes three clusters: class 1 (two molecules, $F_{1}<F_{2}$, left), grouping 2 (three compounds, $F_{1}<<F_{2}$, top) and cluster 3 (four units, $F_{1}>>F_{2}$, right).

Figure 5. $F_{2}$ versus $F_{1}$ scores plot of the principal component analysis for the pesticides.

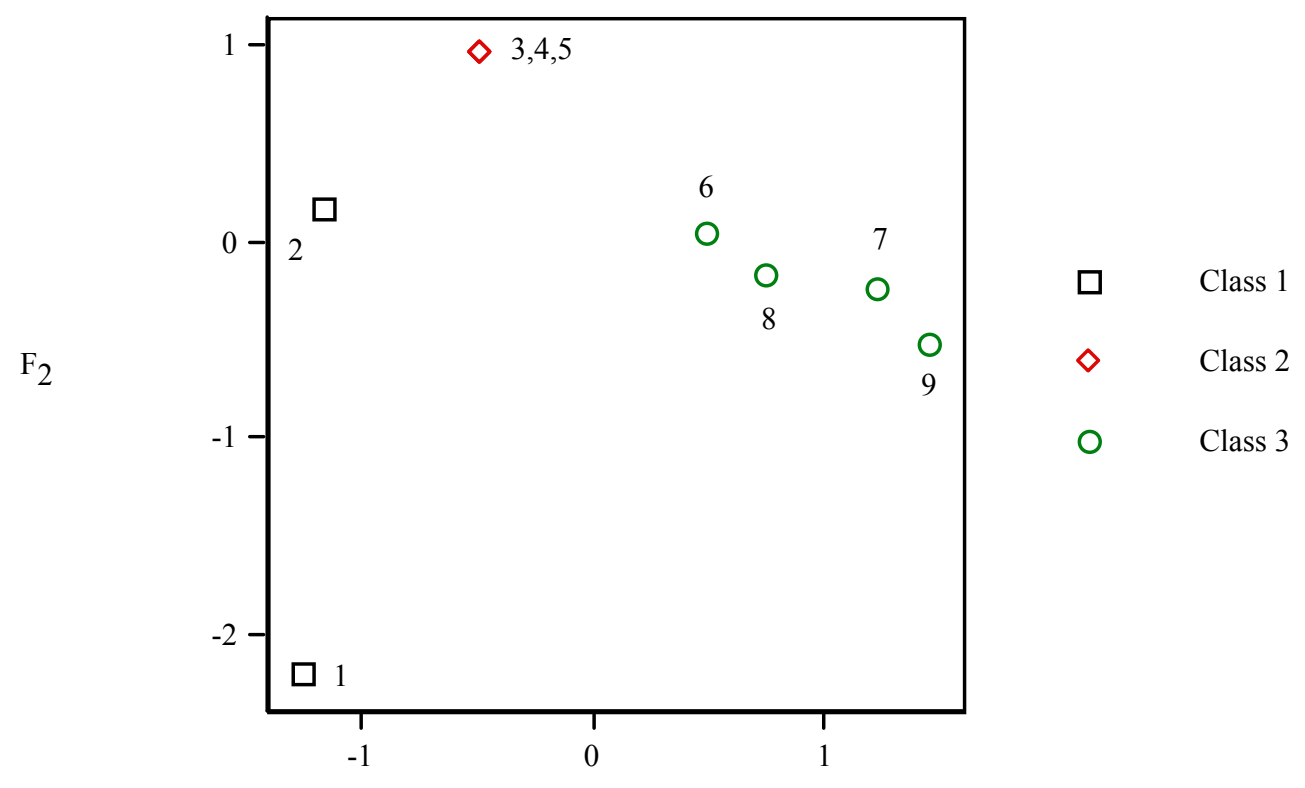

$\mathrm{F}_{1}$

From PCA factor loadings of pesticides, $F_{2}-F_{1}$ loadings plot ( $c f$. Figure 6) depicts the six properties. In addition as a complement to the scores plot (Figure 5) for the loadings (Figure 6), it is confirmed that pesticide 2 located on the left side presents a contribution of cyc $_{123}$ situated near the same side of Figure 5. Class 2 on the top shows more pronounced contribution of $\mathrm{O}_{0345}$ placed in the same position (Figure 6). Two classes of properties are clearly distinguished in the loadings plot: class $1\left\{\mathrm{cyc}_{123}, \mathrm{O}_{0345}, \mathrm{~N}_{13}\right\}\left(F_{1}<F_{2}\right.$, top $)$ and grouping 2 NP,S=,Cl 3$\}\left(F_{1}>>F_{2}\right.$, bottom $)$. 
Figure 6. $F_{2}$ versus $F_{1}$ loadings plot of the principal component analysis for the pesticides.

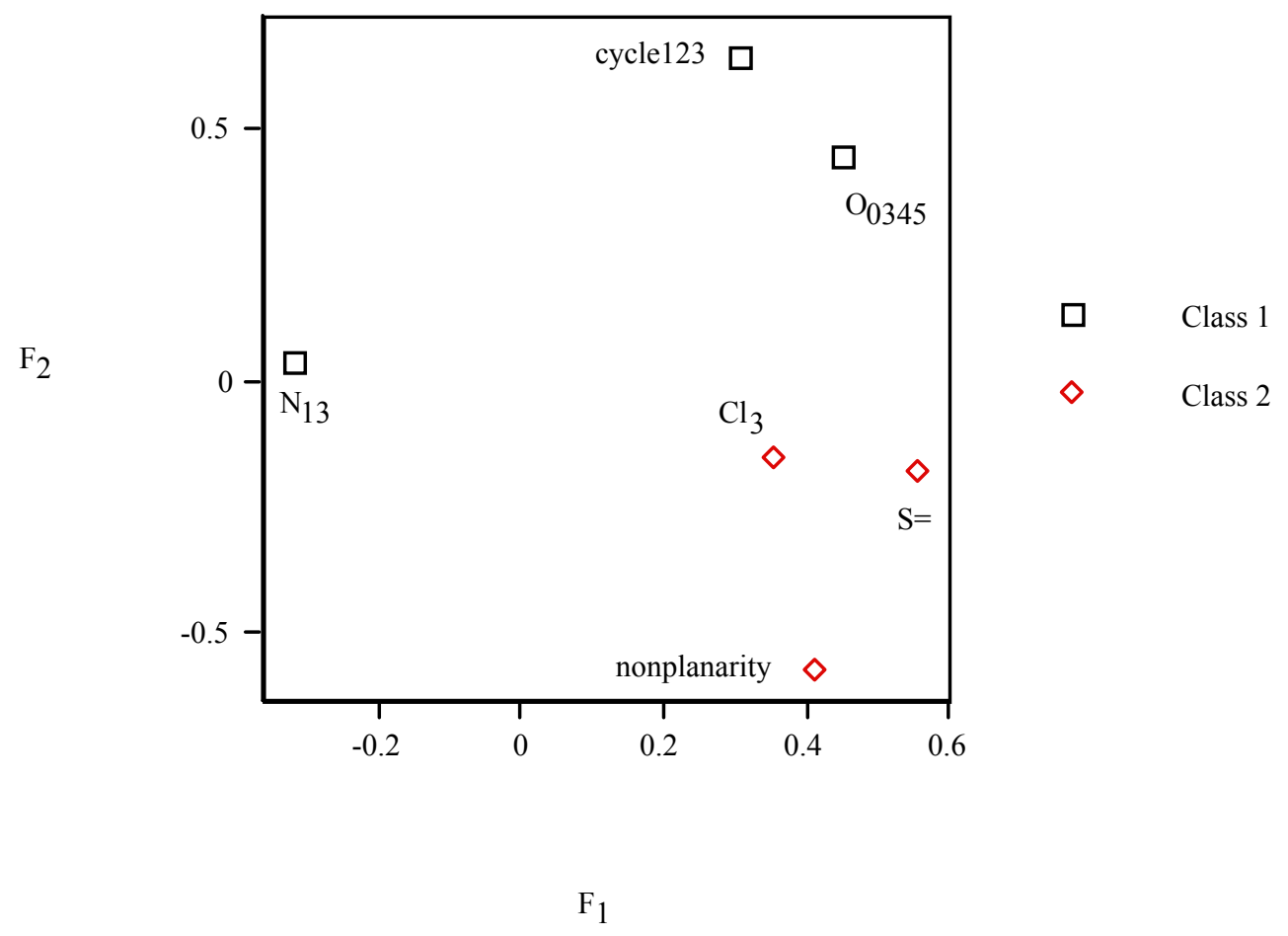

Instead of nine pesticides in the $\mathfrak{R}^{6}$ space of six vector properties, consider six properties in the $\mathfrak{R}^{9}$ space of nine molecules. The upper triangle of matrix $\mathbf{R}$ between pairs of properties resulted in:

$\left.\mathbf{R}=\mid \begin{array}{llllll}0.998 & 0.748 & 0.029 & 0.514 & 0.475 & 0.502 \\ & 0.998 & 0.279 & 0.764 & 0.225 & 0.752 \\ & & 0.998 & 0.482 & 0.506 & 0.471 \\ & & & 0.998 & 0.021 & 0.986 \\ & & & & 0.998 & 0.025 \\ & & & & & 0.998\end{array}\right)$

Correlations are high, e.g., $R_{4,6}=0.986$. Properties dendrogram ( $c f$. Figure 7 ) separates cyc ${ }_{123}$ and $\mathrm{O}_{0345}$ from $\mathrm{N}_{13}$ (class 1), and $\mathrm{Cl}_{3}$ from $\mathrm{NP} / \mathrm{S}=$ (cluster 2) in agreement with PCA loadings plot (Figure 6).

The radial tree for the vector properties ( $c f$. Figure 8 ) separates the same two classes as PCA loadings plot and dendrogram (Figures 6 and 7). Splits graph for properties ( $c f$. Figure 9) reveals conflicting relation between classes because of interdependences. It is in agreement with PCA loadings plot and binary/radial trees (Figures 6-8).

A PCA was performed for the vector properties. Factor $F_{1}$ explains $50 \%$ of variance (50\% error), factors $F_{1 / 2}$ account for $69 \%$ of variance (31\% error), factors $F_{1-3}$ rationalize $82 \%$ of variance (18\% error), etc. In PCA $F_{2}-F_{1}$ scores plot, the same two groupings of properties are distinguished: class 1 $\left\{\right.$ cyc $\left._{123}, \mathrm{O}_{0345}, \mathrm{~N}_{13}\right\} \quad\left(F_{1}>>F_{2}, c f\right.$. Figure 10, right $)$ and grouping $2\left\{\mathrm{NP}, \mathrm{S}=, \mathrm{Cl}_{3}\right\}\left(F_{1}<<F_{2}\right.$, left $)$ in qualitative agreement with PCA loadings plot, binary/radial trees and splits graph (Figures 6-9). 
Figure 7. Dendrogram for the vector properties corresponding to the pesticides.

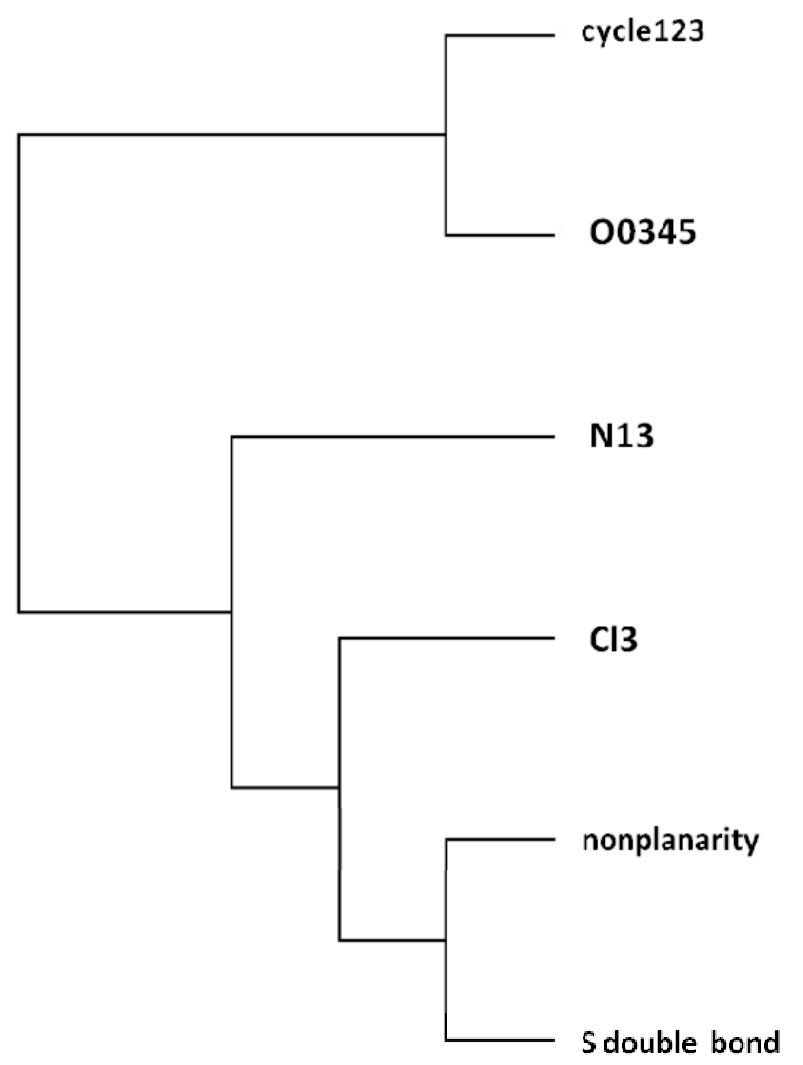

Figure 8. Radial tree for the vector properties corresponding to the pesticides.

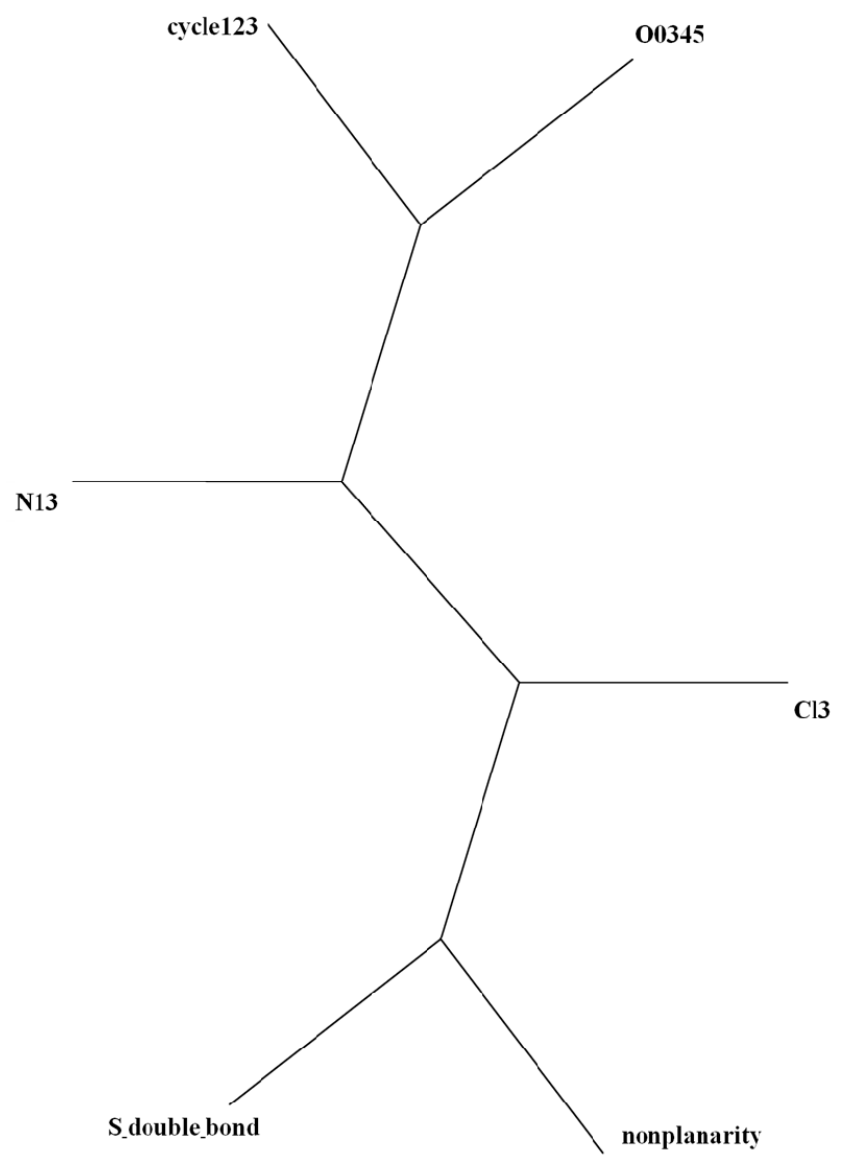


Figure 9. Splits graph for the vector properties corresponding to the pesticides.

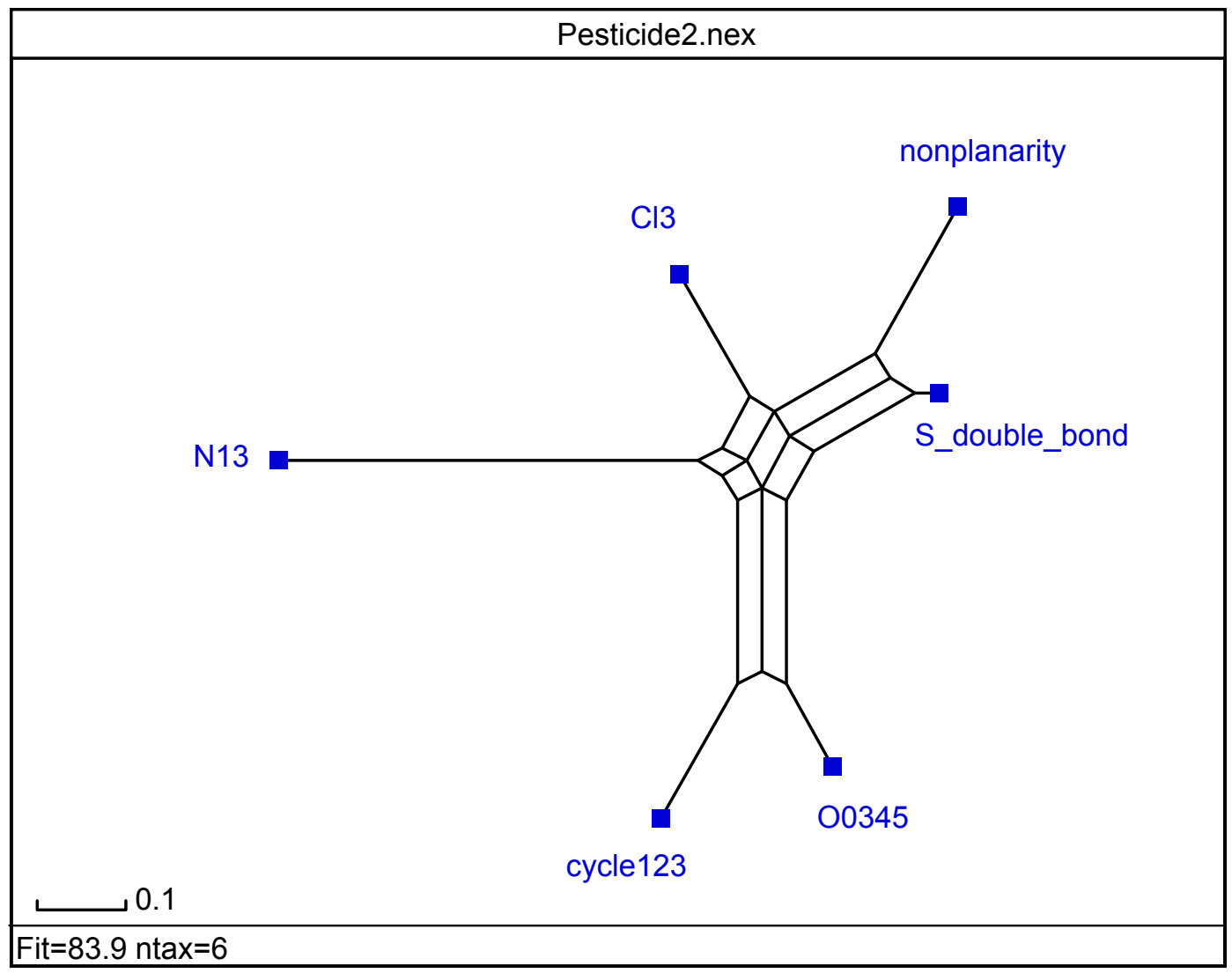

Figure 10. PCA $F_{2}$ vs. $F_{1}$ scores plot for the vector properties corresponding to pesticides.

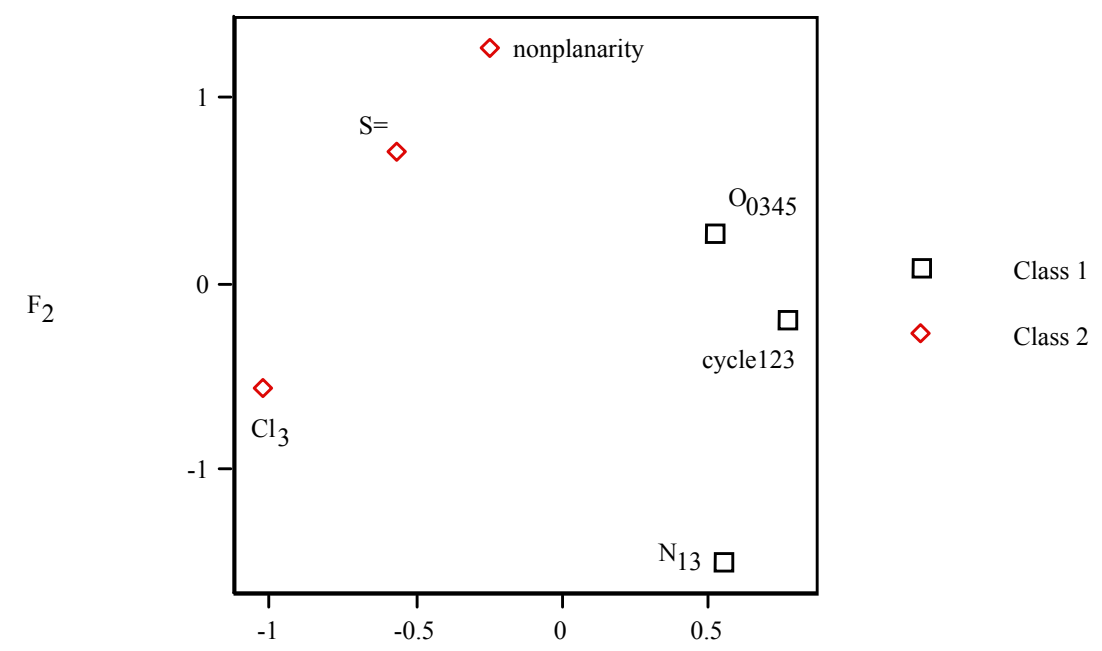

The recommended format of the pesticides periodic table (PT, $c f$. Table 6) shows that they are classified first by $i_{1}, i_{2}, i_{3}, i_{4}, i_{5}$ and, finally, by $i_{6}$. Vertical groups are defined by $\left\langle i_{1}, i_{2}, i_{3}, i_{4}, i_{5}>\right.$ and horizontal periods, by $\left\langle i_{6}>\right.$. Periods of eight units are assumed; e.g., group g00101 stands for $<i_{1}, i_{2}, i_{3}, i_{4}, i_{5}>=<00101>$ : $<001010>\left(\mathrm{cyc}_{0}, \mathrm{O}_{2}, \mathrm{NP}, \mathrm{S}={ }_{0}, \mathrm{~N}_{1}, \mathrm{Cl}_{0}\right)$, etc. Pesticides in the same column appear close in partial correlation diagram, binary/radial trees, splits graph and PCA scores (Figures 1-5). 
Phenylurea herbicides were determined in tap water/soft drink samples by HPLC-UV [49]. Table 6 includes five phenylureas: metazachlor is similar to carbendazim. Can et al. determined sulphonyl/phenylurea herbicides toxicities [50]. Table 6 includes 27 sulphonyl/phenylurea herbicides: (1) phenylureas are similar to metoxuron, monuron, diuron and linuron; (2) sulphonylureas flazasulphuron, triasulphuron, azimsulphuron and chlorsulphuron go with TPP. High-resolution and ultratrace analyses of pesticides were reported via silica $\left(\mathrm{SiO}_{2}\right)$ monoliths [51]. Table 6 takes in six new pesticides: (1) metamitron and phenylurea isoproturon match metoxuron; (2) metolachlor goes with carbendazim; (3) carbofuran agrees with thiabendazole. Qualitative LC-MS analysis of pesticides was informed via monolithic $\mathrm{SiO}_{2}$ capillaries [52]. Table 6 contains two novel pesticides: phenylurea pencycuron tallies metamitron. Analytical standards were provided for persistent organic pollutants (POPs) [53]. Table 6 embraces five POPs: lindane and pentachlorobenzene equal carbetamide

Property $P$ variation of vector $\left\langle i_{1}, i_{2}, i_{3}, i_{4}, i_{5}, i_{6}\right\rangle$ ( $c f$. Figure 11) is expressed in decimal system, $P=10^{5} i_{1}+10^{4} i_{2}+10^{3} i_{3}+10^{2} i_{4}+10 i_{5}+i_{6}$, vs. structural parameters $\left\{i_{1}, i_{2}, i_{3}, i_{4}, i_{5}, i_{6}\right\}$ for the pesticides. Most points and lines $i_{3} / i_{5}$ collapse. For instance, for molecule 1 (methamidophos) $<001010>$, vector property $P=10^{5} \cdot 0+10^{4} \cdot 0+10^{3} \cdot 1+10^{2} \cdot 0+10 \cdot 1+0=1010$ where the structural parameters are 0 and 1 , and the corresponding points are $\left(i_{1}=i_{2}=i_{4}=i_{6}=0, P=1010\right)$ and $\left(i_{3}=i_{5}=1, P=1010\right)$. The results show parameters hierarchy: $i_{1}>i_{2}>i_{3}>i_{4}>i_{5}>i_{6}$ in agreement with PT of properties (Table 6) with vertical groups defined by $\left\{i_{1}, i_{2}, i_{3}, i_{4}, i_{5}\right\}$ and horizontal periods described by $\left\{i_{6}\right\}$. The property was not used in PT development and validates it.

Figure 11. Variation of vector property $P(p)$ of the pesticides versus counts of $\left\{i_{1}, i_{2}, i_{3}\right\}$.

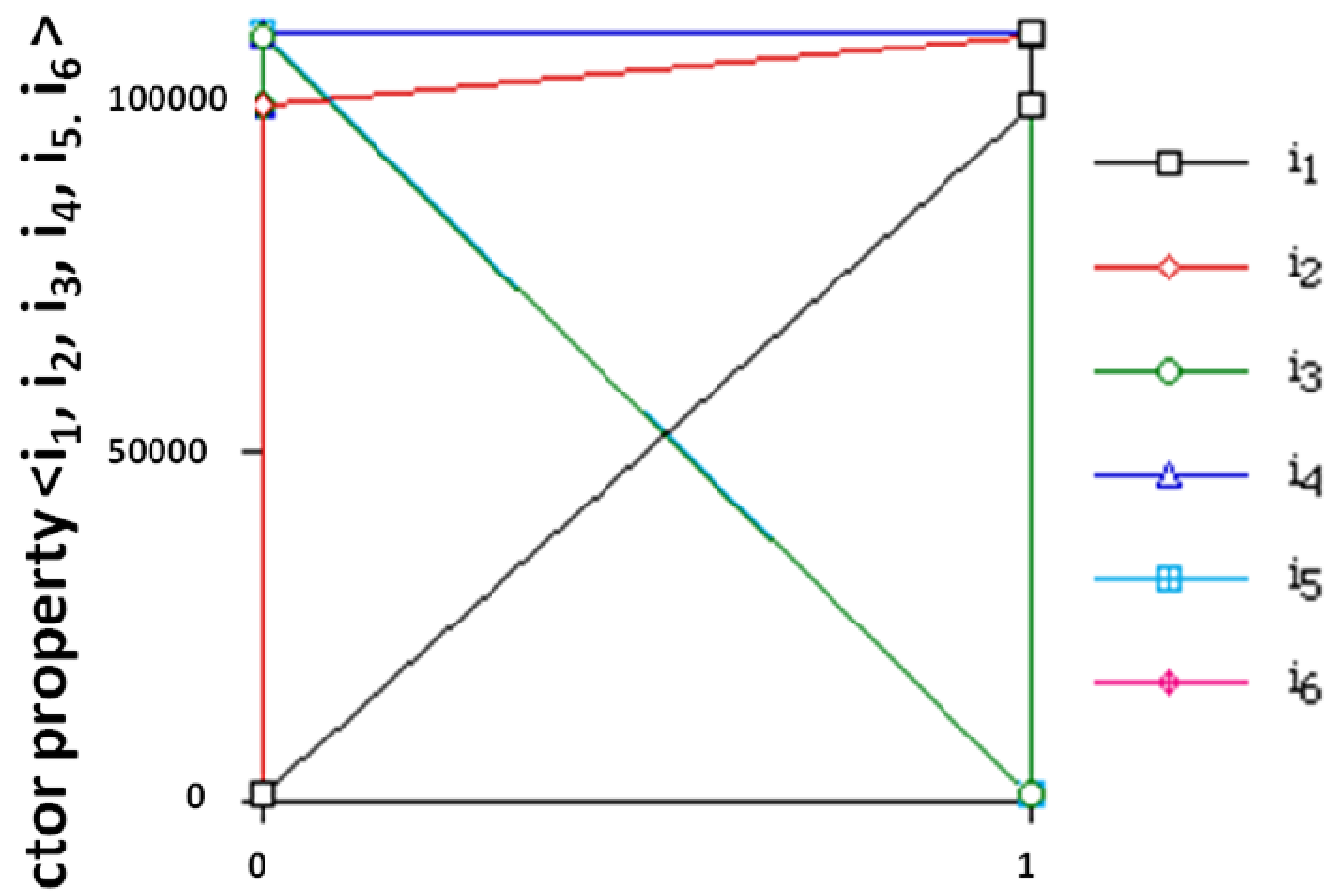

\section{Structural parameter}


Table 6. Table of periodic properties for pesticides, persistent organic pollutants, phenylureas and sulphonylureas.

\begin{tabular}{|c|c|c|c|c|c|c|c|c|c|c|c|}
\hline $\mathbf{P}$. & g00100 & g00101 & g01100 & g10000 & g10001 & g10100 & g11000 & g11001 & g11100 & g11110 & g11111 \\
\hline $\mathrm{p} 0$ & Chlordecone $* *$ & Methamidophos & PFOS ** & $\begin{array}{l}\text { Metamitron * } \\
\text { BDE-99 ** } \\
\text { Metoxuron } \\
\text { Monuron } \\
\text { Diuron } \\
\text { Linuron } \\
\text { Buturon } \\
\text { Chlorotoluron } \\
\text { Daimuron } \\
\text { Fenuron } \\
\text { Methyldimuron } \\
\text { Fluometuron } \\
\text { Siduron } \\
\text { Neburon } \\
\text { Isoproturon } \\
\text { Pencycuron } \\
\end{array}$ & $\begin{array}{c}\text { Carbendazim } \\
\text { Metolachlor * } \\
\text { Metazachlor }\end{array}$ & $\begin{array}{c}\text { AMS } \\
\text { BSM } \\
\text { CME } \\
\text { CNS } \\
\text { EMS } \\
\text { MSM } \\
\text { NCS } \\
\text { OXS } \\
\text { PSE } \\
\text { TFS } \\
\text { TBM } \\
\text { TFO } \\
\text { 3FS } \\
\text { RMS } \\
\text { IDS }\end{array}$ & $\begin{array}{c}\text { Carbetamide * } \\
\text { Prometryne * } \\
\text { Lindane ** } \\
\text { PCB ** }\end{array}$ & $\begin{array}{c}\text { Thiabendazole } \\
\text { Pyrimethanil } \\
\text { Cyprodinil } \\
\text { Carbofuran * }\end{array}$ & $\begin{array}{c}\text { TPP } \\
\text { Flazasulphuron } \\
\text { Triasulphuron } \\
\text { Azimsulphuron } \\
\text { Chlorsulphuron }\end{array}$ & Diazinone & Pyrazophos \\
\hline
\end{tabular}

PFOS: perfluoroctane sulphonate. BDE-99: 2,2',4,4',5-pentabromodiphenylether. AMS: amidosulphuron. BSM: bensulphuron-methyl. CME: Chlorimuron-ethyl. CNS: Cinosulphuron. EMS: ethametsulphuron-methyl. MSM: metsulphuron-methyl. NCS: nicosulphuron. OXS: oxasulphuron. PSE: pyrazosulphuron-ethyl. TFS: Thifensulphuron-methyl. TBM: Tribenuron-methyl. TFO: Trifloxysulphuron-Na. 3FS: triflusulphuron-methyl. RMS: rimsulphuron. IDS: iodosulphuron. PCB: pentachlorobenzene. Regular typeface: pesticides (this work). Regular typeface *: pesticides taken from Ref. [51]. Regular typeface **: persistent organic pollutants. Italics: phenylureas. Bold: sulphonylureas. 
Property $P$ change of vector $\left\langle i_{1}, i_{2}, i_{3}, i_{4}, i_{5}, i_{6}>\right.$ in base 10 ( $c f$. Figure 12) is represented $v s$. number of group in PT, for pesticides (Table 1 subset of Table 6). It reveals minima corresponding to compounds with $<i_{1}, i_{2}, i_{3}, i_{4}, i_{5}>c a$. $<00101>$ (group g00101) and maxima with $<i_{1}, i_{2}, i_{3}, i_{4}, i_{5}>c a .<11111>$ (group g11111). For group 6, period 2 is superimposed on 1. For instance, for group g001010 and period p0, molecule 1 (methamidophos) $<001010>$ lies in the first group in the subset with $P=1010$ and the point is (group $=1, P=1010$ ). Periods $\mathrm{p} 0$ and $\mathrm{p} 1$ represent rows 1 and 2, respectively, in Table 6. Function $P\left(i_{1}, i_{2}, i_{3}, i_{4}, i_{5}, i_{6}\right)$ denotes two periodic waves clearly limited by two maxima, which suggest a periodic behaviour that recalls form of a trigonometric function. For $\left\langle i_{1}, i_{2}, i_{3}, i_{4}, i_{5}, i_{6}\right\rangle$, a maximum is shown. Distance in $<i_{1}, i_{2}, i_{3}, i_{4}, i_{5}, i_{6}>$ units between each pair of consecutive maxima is six, which coincides with pesticide sets in successive periods. The maxima occupy analogous positions and are in phase. The representative points in phase should correspond to elements in the same group in PT. For both maxima, $<i_{1}, i_{2}, i_{3}, i_{4}, i_{5}, i_{6}>$ some coherence exists between two representations; however, the consistency is not general. Waves comparison shows two differences: period 1 is somewhat step-like and period 2 is incomplete. The most characteristic points are maxima, which lie about group g11111. The values of $<i_{1}, i_{2}, i_{3}, i_{4}, i_{5}, i_{6}>$ are repeated as the periodic law (PL) states.

Figure 12. Variation of the vector property $P(p)$ of the pesticides versus group number.

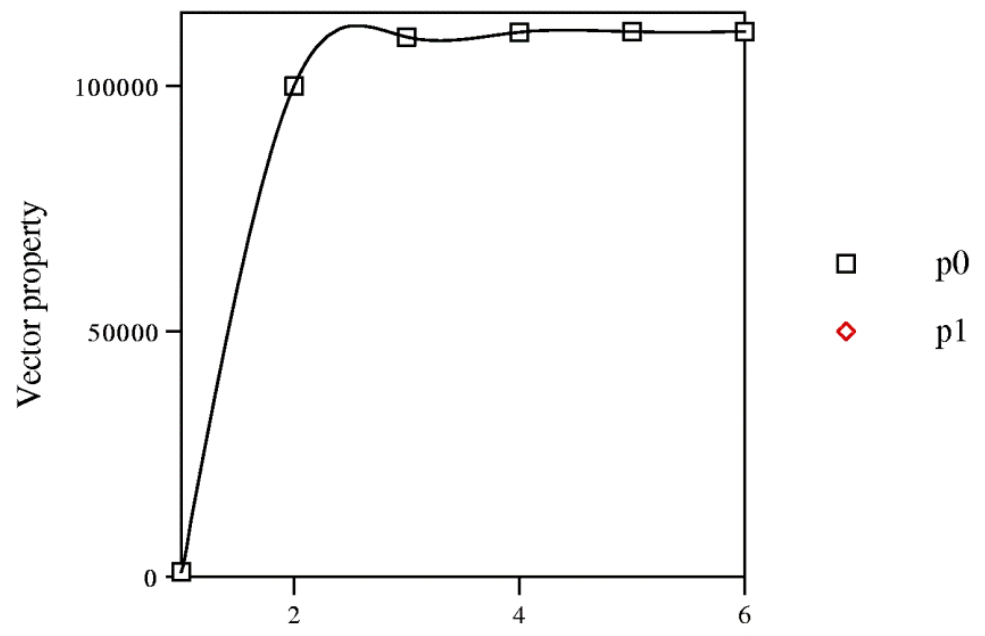

Group number

An empirical function $P(p)$ reproduces the different $<i_{1}, i_{2}, i_{3}, i_{4}, i_{5}, i_{6}>$ values. The minimum of $P(p)$ has meaning only if it is compared with former $P(p-1)$ and later $P(p+1)$ points needing to fulfill:

$$
\begin{aligned}
& P_{\text {min }}(p)<P(p-1) \\
& P_{\text {min }}(p)<P(p+1)
\end{aligned}
$$

The order relations (6) should repeat at determined intervals equal to period size and are equivalent to:

$$
\begin{aligned}
& P_{\min }(p)-P(p-1)<0 \\
& P(p+1)-P_{\text {min }}(p)>0
\end{aligned}
$$


As relations (7) are valid only for minima, more general ones are desired for all values of $p$. Differences $D(p)=P(p+1)-P(p)$ are calculated assigning every value to pesticide $p$ :

$$
D(p)=P(p+1)-P(p)
$$

Instead of $D(p), R(p)=P(p+1) / P(p)$ is taken, assigning them to pesticide $p$. If PL were general, elements in the same group in analogous positions in different periodic waves would satisfy:

$$
\begin{gathered}
\text { either } D(p)>0 \text { or } D(p)<0 \\
\text { and either } R(p)>1 \text { or } R(p)<1
\end{gathered}
$$

However, the results show that this is not the case so that PL is not general, existing some anomalies; e.g., $D(p)$ variation vs. group number ( $c f$. Figure 13) presents lack of coherence between $<i_{1}, i_{2}, i_{3}, i_{4}, i_{5}, i_{6}>$ Cartesian and PT representations. For instance, for group g001010 and period p0, pesticide 1 (methamidophos) $<001010>$ (group $=1, P=1010$ ) presents, in the next PT position, molecule 2 (carbendazim) $<100010>(\mathrm{g} 100010$, group $=2, P=100010), D=100010-1010=99000$ and the point is (group $=1, D=99000$ ). If consistency were rigorous, all points in each period would have the same sign. In general, a trend exists in points to give $D(p)>0$, especially for lower groups.

Figure 13. Variation of property $D(p)=P(p+1)-P(p)$ versus group. $P$ : vector property.

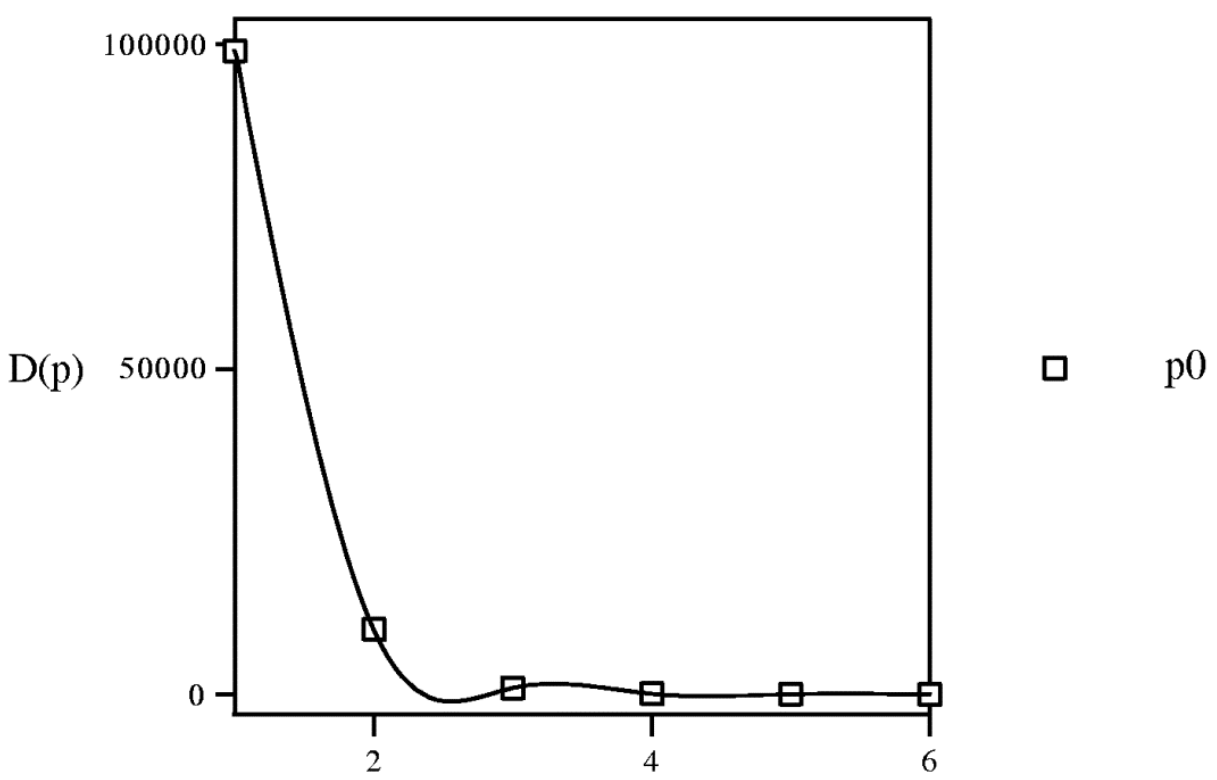

Group number

The change of $R(p) v s$. group number ( $c f$. Figure 14) confirms the lack of constancy between Cartesian and PT charts. For instance, for group g001010 and period p0, pesticide 1 (methamidophos) $<001010>$ (group $=1, P=1010$ ) shows, in the next PT cell, molecule 2 (carbendazim) $<100010>$ (g100010, group $=2, P=100010), R=100010 / 1010=99.0198$ and the point is (group $=1$, $R=99.0198)$. If the steadiness were exact, all points in each period would show $R(p)$ either lesser or greater than one. A trend exists to give $R(p)>1$, especially for the lower groups. 
Figure 14. Variation of property $R(p)=P(p+1) / P(p) v s$. group number. $P$ : vector property.

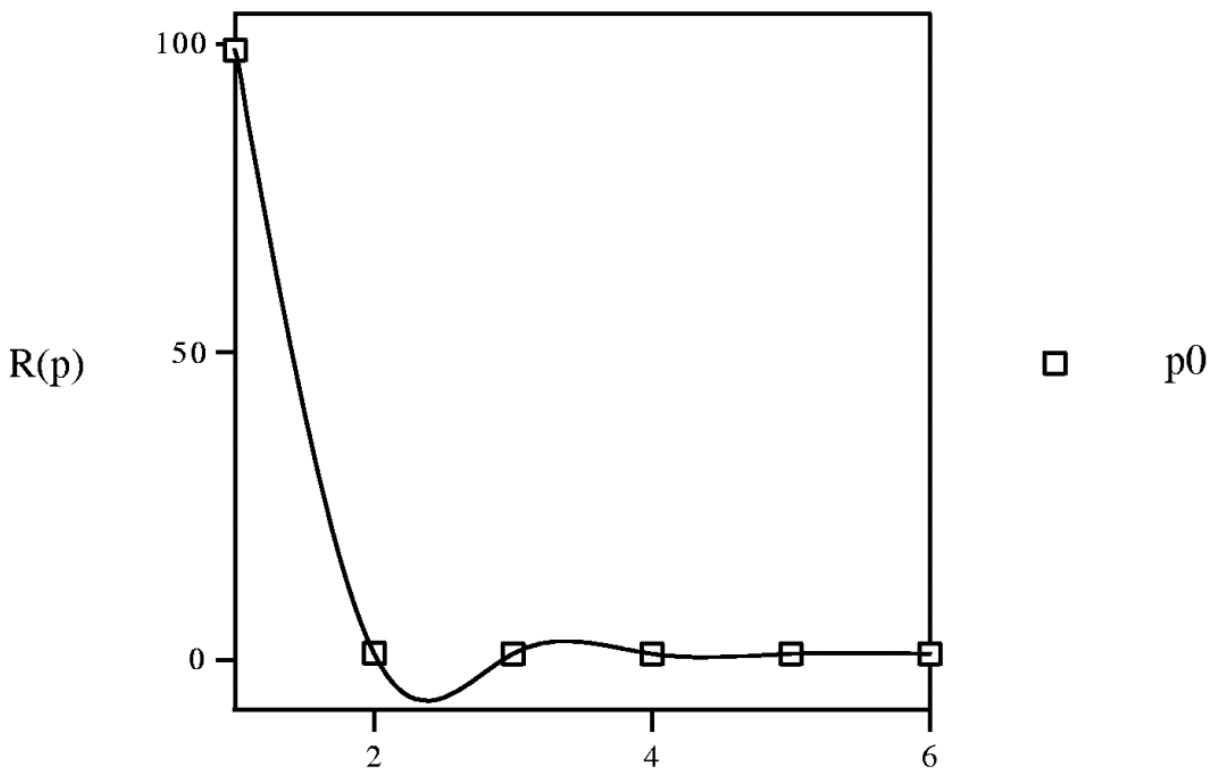

Group number

\section{Experimental}

The key problem in classification studies is to define similarity indices when several criteria of comparison are involved. The first step in quantifying similarity concept for pesticides is to list the most important chemical characteristics of molecules. The vector of properties $\bar{i}=\left\langle i_{1}, i_{2}, \ldots i_{k}, \ldots>\right.$ should be associated with every pesticide $i$, whose components correspond to different molecular features in a hierarchical order according to their expected importance in retention. If characteristic $m$-th is chromatographically more significant for retention than $k$-th then $m<k$. Components $i_{k}$ are either " 1 " or " 0 ", according to whether a similar characteristic of rank $k$ is either present or absent in pesticide $i$ compared to a reference. Analysis includes six structural and constitutional characteristics: presence of cycle $\left(\right.$ cyc $\left._{123}\right)$, occurrence of either none or 3-5 $\mathrm{O}$ atoms $\left(\mathrm{O}_{0345}\right)$, nonplanarity (NP), double-bonded $\mathrm{S}$ atom $(\mathrm{S}=)$, incidence of either one or three $\mathrm{N}$ atoms $\left(\mathrm{N}_{13}\right)$ and existence of three $\mathrm{Cl}$ atoms $\left(\mathrm{Cl}_{3}, c f\right.$. Figure 15). It is assumed that the chemical characteristics can be ranked according to their contribution to retention in the following order of decaying importance: cyc $_{123}>\mathrm{O}_{0345}>\mathrm{NP}>\mathrm{S}=$ $>\mathrm{N}_{13}>\mathrm{Cl}_{3}$. Index $i_{1}=1$ denotes cyc ${ }_{123}\left(i_{1}=0\right.$ for cyc 0$), i_{2}=1$ means $\mathrm{O}_{0345}\left(i_{2}=0\right.$ for $\left.\mathrm{O}_{2}\right), i_{3}=1$ signifies $\mathrm{NP}, i_{4}=1$ indicates $\mathrm{S}=, i_{5}=1$ stands for $\mathrm{N}_{13}\left(i_{5}=0\right.$ for $\mathrm{N}_{0}$ or $\left.\mathrm{N}_{2}\right)$ and $i_{6}=1$ represents $\mathrm{Cl}_{3}\left(i_{6}=0\right.$ for $\left.\mathrm{Cl}_{0}\right)$. In chlorpyrifos number of cycles is one, $\mathrm{O}$ is three, it is $\mathrm{NP}$ and $\mathrm{S}=$, number of $\mathrm{N}$ is one and number of $\mathrm{Cl}$ atoms is three; obviously its associated vector is $<111111>$. In this study chlorpyrifos was selected as reference because of its greatest retention. Table 1 contains vectors associated with nine pesticides. Vector $<001010>$ is associated with methamidophos since it shows $\operatorname{cyc}_{0}, \mathrm{O}_{2}, \mathrm{NP}$, not $\mathrm{S}=, \mathrm{N}_{1}$ and $\mathrm{Cl}_{0}$. 
Let us denote by $r_{i j}\left(0 \leq r_{i j} \leq 1\right)$ similarity index of two pesticides associated with vectors $\bar{i}$ and $\bar{j}$, respectively. Similitude relation is characterized by similarity matrix $\mathbf{R}=\left[r_{i j}\right]$. Similarity index between two pesticides $\bar{i}=<i_{1}, i_{2}, \ldots i_{k} \ldots>$ and $\bar{j}=\left\langle j_{1}, j_{2}, \ldots j_{k} \ldots>\right.$ is defined as:

$$
r_{i j}=\sum_{k} t_{k}\left(a_{k}\right)^{k} \quad(k=1,2, \ldots)
$$

where $0 \leq a_{k} \leq 1$ and $t_{k}=1$ if $i_{k}=j_{k}$ but $t_{k}=0$ if $i_{k} \neq j_{k}$. Definition assigns a weight $\left(a_{k}\right)^{k}$ to any property involved in description of molecule $i$ or $j$.

Figure 15. Pesticides: (a) methamidophos, (b) carbendazim, (c) thiabendazole, (d) pyrimethanil, (e) cyprodinil, (f) diazinone, (g) pyrazophos and (h) chlorpyrifos.<smiles>COP(N)(=O)SC</smiles><smiles>c1ccc2[nH]c(-c3cscn3)nc2c1</smiles><smiles>Cc1cc(C2CC2)nc(Nc2ccccc2)n1</smiles><smiles>CCOC(=O)c1cn2nc(OP(=S)(OCC)OCC)cc2nc1C</smiles><smiles>Cc1cc(C)nc(Nc2ccccc2)n1</smiles><smiles>CCOP(=S)(OCC)Oc1cc(C)nc(C(C)C)n1</smiles>

$\mathrm{h}$<smiles>CCOP(=S)(OCC)Oc1nc(C)c(C)cc1Cl</smiles>

\subsection{Classification Algorithm}

Grouping algorithm uses the stabilized similarity matrix by applying max-min composition rule o:

$$
(\mathbf{R} \circ \mathbf{S})_{i j}=\max _{k}\left[\min _{k}\left(r_{i k}, s_{k j}\right)\right]
$$


where $\mathbf{R}=\left[r_{i j}\right]$ and $\mathbf{S}=\left[s_{i j}\right]$ are matrices of equal type and $(\mathbf{R o S})_{i j}$ is the $(i, j)$-th element of matrix $\mathbf{R o S}$ [54-57]. When applying composition rule max-min iteratively so that $\mathbf{R}(n+1)=\mathbf{R}(n)$ o $\mathbf{R}$, an integer $n$ exists such that: $\mathbf{R}(n)=\mathbf{R}(n+1)=\ldots$ Matrix $\mathbf{R}(n)$ is called stabilized similarity matrix. Its importance lies in fact that in classification it generates partition into disjoint classes. Stabilized matrix is designated by $\mathbf{R}(n)=\left[r_{i j}(n)\right]$. Grouping rule follows: $i$ and $j$ are assigned to the same class if $r_{i j}(n) \geq b$. Class of $i$ noted $\hat{i}$ is set of species $j$ that satisfies rule: $r_{i j}(n) \geq b$. Matrix of classes is:

$$
\widehat{\mathbf{R}}(n)=\left[\widehat{r}_{\hat{i} j}\right]=\max _{s, t}\left(r_{s t}\right) \quad(s \in \hat{i}, t \in \hat{j})
$$

where $s$ stands for any index of species belonging to class $\hat{i}$ (similarly for $t$ and $\hat{j}$ ). Rule (13) means finding largest similarity index between species of two different classes.

\subsection{Information Entropy}

In information theory, information entropy $h$ measures the surprise that source emitting sequences, e.g., cannon-shots, can give $[58,59]$. Consider use of qualitative spot test to determine the presence of $\mathrm{Fe}$ in a water sample. Without any history of testing the analyst must begin by assuming that the two outcomes $0 / 1$ (Fe absent/present) are equiprobable with probabilities $1 / 2$. When up to two metals may be present in sample, e.g., $\mathrm{Fe}$ or $\mathrm{Ni}$, four possible outcomes exist, ranging from neither $(0,0)$ to both present $(1,1)$ with equal probabilities $1 / 4$. Which of four possibilities turns up can be determined via two tests each having two observable states. Similarly with three elements eight possibilities exist each with probability of $1 / 8=1 / 2^{3}$; three tests are needed. Pattern relates uncertainty and information needed to resolve it. Number of possibilities is expressed to power of 2. Power to which 2 must be raised to give number of possibilities $N$ is defined as logarithm to base 2 of that number. Information/uncertainty can be defined in terms of logarithm to base 2 of number of possible analytical outcomes: $I=H=\log _{2} N=\log _{2} 1 / p=-\log _{2} p$, where $I$ is information contained in answer given that $N$ possibilities existed, $H$, initial uncertainty resulting from need to consider $N$ possibilities and $p$, probability of each outcome if all $N$ possibilities are equally likely to occur. The expression is generalized to a situation in which the probability of every outcome is unequal. If one knows from past experience that some elements are more likely to be present than others, the equation is adjusted so that logarithms of individual probabilities suitable weighted are summed: $H=-\Sigma p_{i} \log _{2} p_{i}$, where $\Sigma p_{i}=1$. Consider original example except that now past experience showed that $90 \%$ of samples contained no Fe. Degree of uncertainty is calculated using: $H=-\left(0.9 \log _{2} 0.9+0.1 \log _{2} 0.1\right)=0.469$ bits. For a single event occurring with probability $p$ degree of surprise is proportional to $-\ln p$. Generalizing result to random variable $X$ (which can take $N$ possible values $x_{1}, \ldots, x_{N}$ with probabilities $p_{1}, \ldots, p_{N}$ ) average surprise received on learning $X$ value is: $-\Sigma p_{i} \ln p_{i}$. Information entropy associated with similarity matrix $\mathbf{R}$ is:

$$
h(\mathbf{R})=-\sum_{i, j} r_{i j} \ln r_{i j}-\sum_{i, j}\left(1-r_{i j}\right) \ln \left(1-r_{i j}\right)
$$

Denote by $C_{b}$ set of classes and by $\widehat{\mathbf{R}}_{b}$ similarity matrix at grouping level $b$. Information entropy satisfies following properties. (1) $h(\mathbf{R})=0$ if either $r_{i j}=0$ or $r_{i j}=1$; (2) $h(\mathbf{R})$ is maximum if $r_{i j}=0.5$, 
i.e., when imprecision is maximum; (3) $h\left(\hat{\mathbf{R}}_{b}\right) \leq h(\mathbf{R})$ for any b, i.e., classification leads to entropy loss; (4) $h\left(\hat{\mathbf{R}}_{b_{1}}\right) \leq h\left(\hat{\mathbf{R}}_{b_{2}}\right)$ if $b_{1}<b_{2}$, i.e., entropy is monotone function of grouping level $b$.

\subsection{Equipartition Conjecture of Entropy Production}

In classification algorithm every hierarchical tree corresponds to entropy dependence on grouping level and diagram $h-b$ is obtained. Tondeur and Kvaalen equipartition conjecture of entropy production is proposed as selection criterion among hierarchical trees. According to conjecture for given charge, dendrogram (binary tree) with best configuration is that in which entropy production is most uniformly distributed. One proceeds by analogy using information instead of thermodynamic one. Equipartition implies linear dependence so that equipartition line results:

$$
h_{\text {eqp }}=h_{\text {max }} b
$$

Since classification is discrete, way of expressing equipartition would be regular staircase function. Best variant is chosen to be that minimizing sum of squares of deviations:

$$
S S=\sum_{b_{i}}\left(h-h_{\text {eqp }}\right)^{2}
$$

\subsection{Learning Procedure}

Learning procedures were implemented similar to those encountered in stochastic methods [60]. Consider a given partition into classes as good from practical observations, which corresponds to reference similarity matrix $\mathbf{S}=\left[s_{i j}\right]$ obtained for equal weights $a_{1}=a_{2}=\ldots=a$ and for an arbitrary number of fictious properties. Next consider the same set of species as in the good classification and actual properties. Degree of similarity $r_{i j}$ is computed with Equation (11) giving matrix R. Number of properties for $\mathbf{R}$ and $\mathbf{S}$ differs. Learning procedure consists in finding classification results for $\mathbf{R}$ as close as possible to good classification. First weight $a_{1}$ is taken constant and only following weights $a_{2}$, $a_{3}, \ldots$ are subjected to random variations. New similarity matrix is obtained using Equation (11) and new weights. Distance between partitions into classes characterized by $\mathbf{R}$ and $\mathbf{S}$ is:

$$
D=-\sum_{i j}\left(1-r_{i j}\right) \ln \frac{1-r_{i j}}{1-s_{i j}}-\sum_{i j} r_{i j} \ln \frac{r_{i j}}{s_{i j}} \quad\left(\forall 0 \leq r_{i j}, s_{i j} \leq 1\right)
$$

Definition was suggested by that introduced in information theory to measure distance between two probability distributions [61]. In the present case it is distance between matrices $\mathbf{R}$ and $\mathbf{S}$. Since for every matrix a corresponding classification exists two classifications will be compared by distance, which is nonnegative quantity that approaches zero as resemblance between $\mathbf{R}$ and $\mathbf{S}$ increases. The algorithm result is a set of weights allowing classification. The procedure was applied to the synthesis of complex dendrograms using information entropy [62-65]. Our program MolClas is simple, reliable, efficient and fast procedure for molecular classification, based on equipartition conjecture of entropy production according to Equations (11)-(17); it reads number of properties and molecular properties; it allows optimization of coefficients; it optionally reads starting coefficients and number of iteration cycles. Correlation matrix can be either calculated by program or read from input file. The MolClas calculates property similarity matrix in symmetric storage mode; it applies graphical correlation model 
for partial correlation diagram; it computes classifications, calculates distances between clusters, computes groupings similarity matrices, works out classifications information entropy, optimizes coefficients, performs single/complete-linkage hierarchical cluster analyses and plots cluster diagrams; it was written not only to analyze the equipartition conjecture of entropy production but also to explore the world of molecular classification.

\section{Conclusions}

From the present results and discussion the following conclusions can be drawn:

(1) The objective was to develop a structure-property relation for qualitative and quantitative prediction of chromatographic retention times of pesticides. Results of the present work contribute to relation prediction of pesticide residues, in food and environmental samples. Code TOPO allows fractal dimensions, and SCAP, solvation free energies and partition coefficient, which show that for a given atom energies and partitions are sensitive to the presence in the molecule of other atoms and functional groups. Fractal dimensions, partition coefficient, etc. differentiated pesticides. Parameters needed for co-ordination index are molar formation enthalpy, molecular weight and surface area. The morphological and co-ordination indices barely improved equations. Correlation between molecular area and weight points not only to a homogeneous molecular structure of pesticides, but also to the ability to predict and tailor their properties; the latter is nontrivial in environmental toxicology.

(2) Several criteria selected to reduce the analysis to a manageable quantity of pesticides, referred to structural and constituent characteristics related to nonplanarity, and the number of rings, and $\mathrm{O}$, double-bonded $\mathrm{S}, \mathrm{N}$ and $\mathrm{Cl}$ atoms. Classification was in agreement with the principal component analyses. Program MolClas is a simple, reliable, efficient and fast procedure for molecular classification based on equipartition conjecture of entropy production. It was written to analyze equipartition conjecture of entropy production and explore molecular-classification world.

(3) Periodic law does not satisfy physics-law status: (a) pesticides retentions are not repeated; perhaps chemical character; (b) order relations are repeated with exceptions. Analysis forces statement: Relations that any compound $p$ has with its neighbour, $p+1$, are approximately repeated for each period. Periodicity is not general; however, if substance natural order is accepted law must be phenomenological. Retention is not used in periodictable generation and serves to validate it. The analysis of other properties would give an insight into the possible generality of the periodic table. The periodic classification was extended to phenylureas and sulphonylureas.

\section{Acknowledgments}

This work was funded by the own resources of our universities. 


\section{Author Contributions}

Both authors designed and performed research, and analyzed the data; FT wrote the report. Both authors read and approved the final manuscript.

\section{Conflicts of Interest}

The authors declare no conflict of interest.

\section{References}

1. De Melo Abreu, S.; Caboni, P.; Cabras, P.; Garau, V.L.; Alves, A. Validation and global uncertainty of a liquid chromatographic with diode array detection method for the screening of azoxystrobin, kresoxim-methyl, trifloxystrobin, famoxadone, pyraclostrobin and fenamidone in grapes and wine. Anal. Chim. Acta 2006, 291, 573-574.

2. Oliva, J.; Navarro, S.; Barba, A.; Navarro, G. Determination of chlorpyrifos, penconazole, fenarimol, vinclozolin and metalaxyl in grapes, must and wine by on-line microextraction and gas chromatography. J. Chromatogr. A 1999, 833, 43-51.

3. Jiménez, J.J.; Bernal, J.L.; del Nozal, M.J.; Toribio, L.; Arias, E. Analysis of pesticide residues in wine by solid-phase extraction and gas chromatography with electron capture and nitrogen-phosphorus detection. J. Chromatogr. A 2001, 919, 147-156.

4. Wang, J.F.; Luan, L.; Wang, Z.Q.; Jiang, S.R.; Pan, C.P. Determination of 19 multi-residue pesticides in grape wine by gas chromatography-mass spectrometry with micro liquid-liquid extraction and solid phase extraction. Chin. J. Anal. Chem. 2007, 35, 1430-1434.

5. Economou, A.; Botitsi, H.; Antoniou, S.; Tsipi, D. Determination of multi-class pesticides in wines by solid-phase extraction and liquid chromatography-tandem mass spectrometry. J. Chromatogr. A 2009, 1216, 5856-5867.

6. Hu, Y.; Liu, W.M.; Zhou, Y.M.; Guan, Y.F. Determination of organophosphorous pesticide residues in red wine by solid phase microextraction-gas chromatography. Chin. J. Chromatogr. 2006, 24, 290-293.

7. Wu, J.; Tragas, C.; Lord, H.; Pawliszyn, J. Analysis of polar pesticides in water and wine samples by automated in-tube solid-phase microextraction coupled with high-performance liquid chromatography-mass spectrometry. J. Chromatogr. A 2002, 976, 357-367.

8. Bolaños, P.P.; Romero-González, R.; Frenich, A.G.; Vidal, J.L.M. Application of hollow fibre liquid phase microextraction for the multiresidue determination of pesticides in alcoholic beverages by ultra-high pressure liquid chromatography coupled to tandem mass spectrometry. J. Chromatogr. A 2008, 1208, 16-24.

9. Vinas, P.; Aguinaga, N.; Campillo, N.; Hernández-Córdoba, M. Comparison of stir bar sorptive extraction and membrane-assisted solvent extraction for the ultra-performance liquid chromatographic determination of oxazole fungicide residues in wines and juices. J. Chromatogr. A 2008, 1194, $178-183$. 
10. Anastassiades, M.; Lehotay, S.J.; Stajnbaher, D.; Schenck, F.J. Fast and easy multiresidue method employing acetonitrile extraction/partitioning and "dispersive solid-phase extraction" for the determination of pesticide residues in produce. J. AOAC Int. 2003, 86, 412-431.

11. Lehotay, S.J. Mass Spectrometry in Food Safety; Zweigenbaum, J., Ed.; Methods in Molecular Biology No. 747; Humana: Totowa, NJ, USA, 2011; pp. 65-91.

12. Cunha, S.C.; Lehotay, S.J.; Mastovska, K.; Fernandes, J.O.; Beatriz, M.; Oliveira, P.P. Evaluation of the QuEChERS sample preparation approach for the analysis of pesticide residues in olives. J. Sep. Sci. 2007, 30, 620-632.

13. Whelan, M.; Kinsella, B.; Furey, A.; Moloney, M.; Cantwell, H.; Lehotay, S.J.; Danaher, M. Determination of anthelmintic drug residues in milk using ultra high performance liquid chromatography-tandem mass spectrometry with rapid polarity switching. J. Chromatogr. A 2010, 1217, 4612-4622.

14. Wang, X.; Telepchak, M.J. Determination of pesticides in red wine by QuEChERS extraction, rapid mini-cartridge cleanup and LC-MS-MS detection. LC.GC Eur. 2013, 26, 66-76.

15. Blasco, C.; Picó, Y. Prospects for combining chemical and biological methods for integrated environmental assessment. Trends Anal. Chem. 2009, 28, 745-757.

16. Guia de Potabilidade para Substàncias Químicas; de Umbuzeiro, A.G., Ed.; Limiar: São Paulo, Brazil, 2012. Available online: http://www.abes-sp.org.br/arquivos/ctsp/guia_potabilidade.pdf (accessed on 5 June 2014).

17. Moganti, S.; Richardson, B.J.; McClellan, K.; Martin, M.; Lam, P.K.S.; Zheng, G.J. Use of the clam Asaphis deflorata as a potential indicator of organochlorine bioaccumulation in Hong Kong coastal sediments. Mar. Pollut. Bull. 2008, 57, 672-680.

18. Barco-Bonilla, N.; Romero-González, R.; Plaza-Bolaños, P.; Garrido Frenich, A.; Martínez Vidal, J.L. Analysis and study of the distribution of polar and non-polar pesticides in wastewater effluents from modern and conventional treatments. J. Chromatogr. A 2010, 1217, 7817-7825.

19. Navarro, A.; Tauler, R.; Lacorte, S.; Barceló, D. Chemometrical investigation of the presence and distribution of organochlorine and polyaromatic compounds in sediments of the Ebro River Basin. Anal. Bioanal. Chem. 2006, 385, 1020-1030.

20. Navarro-Ortega, A.; Tauler, R.; Lacorte, S.; Barceló, D. Occurrence and transport of PAHs, pesticides and alkylphenols in sediment samples along the Ebro River Basin. J. Hydrol. 2010, 383, 5-17.

21. Toropov, A.A.; Toropova, A.P.; Benfenati, E. QSPR modelling of the octanol/water partition coefficient of organometallic substances by optimal SMILES-based descriptors. Cent. Eur. J. Chem. 2009, 7, 846-856.

22. Toropova, A.P.; Toropov, A.A.; Benfenati, E.; Gini, G. QSAR models for toxicity of organic substances to Daphnia magna built up by using the CORAL freeware. Chem. Biol. Drug Des. 2011, 79, 332-338.

23. Toropov, A.A.; Toropova, A.P. Optimal descriptor as a translator of eclectic data into endpoint prediction: Mutagenicity of fullerene as a mathematical function of conditions. Chemosphere 2014, 104, 262-264. 
24. Kar, S.; Roy, K. Predictive chemometric modeling and three-dimensional toxicophore mapping of diverse organic chemicals causing bioluminescent repression of the bacterium genus Pseudomonas. Ind. Eng. Chem. Res. 2013, 52, 17648-17657.

25. Roy, K.; Das, R.N.; Popelier, P.L.A. Quantitative structure-activity relationship for toxicity of ionic liquids to Daphnia magna: Aromaticity vs. lipophilicity. Chemosphere 2014, 112, 120-127.

26. Torrens, F. Free energy of solvation and partition coefficients in methanol-water binary mixtures. Chromatographia 2001, 53, S199-S203.

27. Soria, V.; Campos, A.; Figueruelo, J.E.; Gómez, C.; Porcar, I.; García, R. Modelling of stationary phase in size-exclusion chromatography with binary eluents. In Strategies in Size Exclusion Chromatography; Potschka, M., Dubin, P.L., Eds.; ACS Symposium Series. No. 635; American Chemical Society: Washington, DC, USA, 1996; Chapter 7, pp. 103-126.

28. Torrens, F.; Soria, V. Stationary-mobile phase distribution coefficient for polystyrene standards. Sep. Sci. Technol. 2002, 37, 1653-1665.

29. Torrens, F. A new chemical index inspired by biological plastic evolution. Indian J. Chem. Sect. A 2003, 42, 1258-1263.

30. Torrens, F. A chemical index inspired by biological plastic evolution: Valence-isoelectronic series of aromatics. J. Chem. Inf. Comput. Sci. 2004, 44, 575-581.

31. Torrens, F.; Castellano, G. QSPR prediction of retention times of phenylurea herbicides by biological plastic evolution. Curr. Drug Saf. 2012, 7, 262-268.

32. Torrens, F.; Castellano, G. Molecular categorization of phenylurea and sulphonylurea herbicides, pesticides and persistent organic pollutants. In QSAR in Drug and Environmental Research; Roy, K., Ed.; IGI Global: Hershey, PA, USA, 2015; in press.

33. Torrens, F.; Castellano, G. QSPR prediction of chromatographic retention times of pesticides: Partition and fractal indices. J. Environ. Sci. Health Part B 2014, 49, 400-407.

34. Varmuza, K. Pattern Recognition in Chemistry; Springer: New York, NY, USA, 1980.

35. Benzecri, J.P. L'Analyse des Données; Dunod: Paris, France, 1984; Volume 1.

36. Tondeur, D.; Kvaalen, E. Equipartition of entropy production. An optimality criterion for transfer and separation processes. Ind. Eng. Chem. Fundam. 1987, 26, 50-56.

37. Torrens, F. Characterizing cavity-like spaces in active-site models of zeolites. Comput. Mater. Sci. 2003, 27, 96-101.

38. IMSL. Integrated Mathematical Statistical Library (IMSL); IMSL: Houston, TX, USA, 1989.

39. Tryon, R.C. A multivariate analysis of the risk of coronary heart disease in Framingham. J. Chronic Dis. 1939, 20, 511-524.

40. Jarvis, R.A.; Patrick, E.A. Clustering using a similarity measure based on shared nearest neighbors. IEEE Trans. Comput. 1973, C22, 1025-1034.

41. Page, R.D.M. Program TreeView; Universiy of Glasgow: Glasgow, UK, 2000.

42. Huson, D.H. SplitsTree: Analizing and visualizing evolutionary data. Bioinformatics 1998, 14, $68-73$.

43. Hotelling, H. Analysis of a complex of statistical variables into principal components. J. Educ. Psychol. 1933, 24, 417-441.

44. Kramer, R. Chemometric Techniques for Quantitative Analysis; Marcel Dekker: New York, NY, USA, 1998. 
45. Patra, S.K.; Mandal, A.K.; Pal, M.K. State of aggregation of bilirubin in aqueous solution: Principal component analysis approach. J. Photochem. Photobiol. A 1999, 122, 23-31.

46. Jolliffe, I.T. Principal Component Analysis; Springer: Berlin, Germany, 2002.

47. Xu, J.; Hagler, A. Chemoinformatics and drug discovery. Molecules 2002, 7, 566-600.

48. Shaw, P.J.A. Multivariate Statistics for the Environmental Sciences; Hodder-Arnold: New York, NY, USA, 2003.

49. Kaur, M.; Malik, A.K.; Singh, B. Determination of phenylurea herbicides in tap water and soft drink samples by HPLC-UV and solid-phase extraction. LC.GC Eur. 2012, 25, 120-129.

50. Can, A.; Yildiz, I.; Guvendik, G. The determination of toxicities of sulphonylurea and phenylurea herbicides with quantitative structure-toxicity relationship (QSTR) studies. Environ. Toxicol. Pharmacol. 2013, 35, 369-379.

51. Cabrera, K.; Altmaier, S. High-resolution and ultra trace analysis of pesticides using silica monoliths. Int. Labmate 2013, 38, 4-5.

52. Forster, S.; Altmaier, S. Qualitative LC-MS analysis of pesticides using monolithic silica capillaries and potential for assay of pesticides in kidney. LC.GC Eur. 2013, 26, 488-496.

53. Nold, M. Analytical standards for persistent organic pollutants. Analytix 2009, 2009, 11-12.

54. Kaufmann, A. Introduction à la Théorie des Sous-ensembles Flous; Masson: Paris, France, 1975; Volume 3.

55. Cox, E. The Fuzzy Systems Handbook; Academic: New York, NY, USA, 1994.

56. Kundu, S. The min-max composition rule and its superiority over the usual max-min composition rule. Fuzzy Sets Sys. 1998, 93, 319-329.

57. Lambert-Torres, G.; Pereira Pinto, J.O.; Borges da Silva, L.E. Minmax techniques. In Wiley Encyclopedia of Electrical and Electronics Engineering; Wiley: New York, NY, USA, 1999.

58. Shannon, C.E. A mathematical theory of communication: Part I, discrete noiseless systems. Bell Syst. Tech. J. 1948, 27, 379-423.

59. Shannon, C.E. A mathematical theory of communication: Part II, the discrete channel with noise. Bell Syst. Tech. J. 1948, 27, 623-656.

60. White, H. Neural network learning and statistics. AI Expert 1989, 4, 48-52.

61. Kullback, S. Information Theory and Statistics; Wiley: New York, NY, USA, 1959.

62. Iordache, O.; Corriou, J.P.; Garrido-Sánchez, L.; Fonteix, C.; Tondeur, D. Neural network frames. application to biochemical kinetic diagnosis. Comput. Chem. Eng. 1993, 17, 1101-1113.

63. Iordache, O. Modeling Multi-Level Systems; Springer: Berlin, Germany, 2011.

64. Iordache, O. Self-Evolvable Systems: Machine Learning in Social Media; Springer: Berlin, Germany, 2012.

65. Iordache, O. Polytope Projects; CRC: Boca Raton, FL, USA, 2014.

Sample Availability: Not available.

(C) 2014 by the authors; licensee MDPI, Basel, Switzerland. This article is an open access article distributed under the terms and conditions of the Creative Commons Attribution license (http://creativecommons.org/licenses/by/3.0/). 